\title{
PRICING OF CYBER INSURANCE CONTRACTS IN A NETWORK MODEL
}

\author{
BY \\ Matthias A. FAhrenwaldt, Stefan Weber And Kerstin Weske
}

\begin{abstract}
We develop a novel approach for pricing cyber insurance contracts. The considered cyber threats, such as viruses and worms, diffuse in a structured data network. The spread of the cyber infection is modeled by an interacting Markov chain. Conditional on the underlying infection, the occurrence and size of claims are described by a marked point process. We introduce and analyze a new polynomial approximation of claims together with a mean-field approach that allows to compute aggregate expected losses and prices of cyber insurance. Numerical case studies demonstrate the impact of the network topology and indicate that higher order approximations are indispensable for the analysis of non-linear claims.
\end{abstract}

\section{KEYWORDS}

Cyber insurance, emerging risks, polynomial approximation, mean-field approximation.

\section{INTRODUCTION}

Cyber risk has evolved as a major threat to businesses. For instance, Lloyd's of London estimates that the total extent of cyber attacks to businesses worldwide comprises losses of USD 400 billion a year (Gandel, 2015). In addition, the size of exposure to cyber risks might significantly grow in our interconnected world. Although companies seem aware of these threats, recent studies find that relatively few firms have yet built a formal cyber risk management system (Swiss Re/IBM, 2016). Their management of cyber risks consists mostly of ad-hoc selfprotection mechanisms such as firewalls and anti-virus software, but very few perform regular cyber risk assessments and possess risk management programs that integrate cyber risk on an institutional level.

Cyber damage may occur accidentally, but might also be purposely caused. As described in Swiss Re/IBM (2016), costs "are no longer confined to coping with lost, stolen or corrupted data, but increasingly include potential damage 
to a firm's property and reputation, and also the cost associated with business interruption or severe disruption to critical infrastructure". Even if future risk management strategies were able to actively improve protection against the occurrence or impact of cyber events, some residual cyber risks would remain that require insurance solutions.

From an actuarial point of view, cyber risk is challenging in three ways. First, data is not available in the required amount or in the desired granularity to apply standard statistical methods. Second, technology and cyber threats are evolving fast; the cyber environment is highly non-stationary. Third, infectious cyber threats pose a large accumulation risk to an insurance company. The typical insurance independence assumption does not hold and, moreover, there is no geographical distinction between dependent groups as there is, for example, for natural catastrophes. This requires new mathematical models that capture the dependence structure of cyber networks in an appropriate way (see also Swiss Re Institute, 2017).

This is the aim of the present paper. Causes and channels for cyber losses are diverse. While an insider attack might cause substantial damage to a particular firm, it might not affect other firms. However, if the originally attacked firm is part of a larger network in which the operations of components depend on each other, consecutive and coupled losses might occur. Another related example are worms, viruses and Trojans that spread across data networks and facilitate attacks throughout the network. Our paper focuses on cyber threats in networks, i.e., infectious cyber threats.

From an insurance perspective, our main contributions are the following:

i. To the best of our knowledge, we develop the first mathematical model of insured losses generated by infectious cyber threats. Dependence is modeled as an undirected network where each node could represent a firm, a system of computers or a single device; each edge constitutes a possible transmission channel in a network.

ii. We provide a new methodology to calculate expected aggregate losses of a (re-)insurance company. Our method is applicable to a large variety of contract designs including both linear and non-linear functions of the generated losses. The application is illustrated in numerical examples. Thus, our approach can be used for pricing decisions.

iii. In numerical case studies, we analyze the role of the network topology. We find that the insured network structure has a significant impact on the generated losses. This illustrates that the topology of the network is a key ingredient for the pricing of cyber insurance contracts and for cyber risk management in general.

Our model consists of two parts: a stochastic process that captures cyber infections, and a mechanism that randomly generates the actual claims at the infected nodes. To model the claims, we introduce a marked point process. This is in spirit of the collective risk model of insurance (see, e.g., Burnecki et al., 2011). In contrast to the standard setting, the claims in our model depend on the spread 
of the cyber threat. The cyber infections are modeled by a susceptible-infectedsusceptible (SIS) network process (see, e.g., Pastor-Satorras et al., 2015, Section $\mathrm{V})$. For a network of size $N$, this approach results in a continuous-time Markov chain with a very large state space of size $2^{N}$. In order to cope with the resulting computational challenges, we develop a tractable mean-field approximation for the Markov process. Approximations of its moments are derived as solutions to systems of ordinary differential equations (ODEs) (see, e.g., Van Mieghem et al., 2009 and Van Mieghem, 2011). Combining these solutions with our claim model, finally, yields approximations of the expected insured losses. For this purpose, we develop a polynomial approximation approach to evaluate general, possibly non-linear, claim functions. In the context of SIS-processes, we provide the following contributions to the theory of mean-field approximations:

i. We suggest a general and rigorous framework for mean-field approximations of the moments of the spread process of arbitrary order $n \geq 1$. We show that there are two key ingredients defining the approximation: a meanfield function, and a splitting algorithm.

ii. We analyze two mean-field functions in detail that lead to different schemes: the well-known independent approximation (also called NIMFA approximation, see Van Mieghem et al., 2009) and a new approximation type: the Hilbert approximation.

iii. For the first-order independent approximation, we derive a time-dependent accuracy result.

iv. For both approximation types, NIMFA and Hilbert, we provide splitting algorithms and briefly address the question of splitting.

\subsection{Literature}

The paper connects three different research areas: mathematical modeling of cyber insurance, epidemics on networks and marked point processes.

1.1.1. Cyber insurance. The literature on mathematical cyber insurance modeling focuses mainly on simple game-theoretic models. These consider, e.g., the following questions: Does a cyber insurance market exist in equilibrium (Böhme, 2005)? Does cyber insurance affect the incentives to self-protection (Bolot and Lelarge, 2008) and how does it influence social welfare (Schwartz and Sastry, 2014)? A recent review of such game-theoretic approaches can be found in the survey article Marotta et al. (2015). In contrast to these papers, we model cyber insurance with the aim of simulating and evaluating losses and pricing insurance contracts. To date, cyber loss models are mostly based on deterministic scenarios (see Swiss Re Institute, 2017). In contrast, we derive a stochastic model and develop suitable approximation techniques to explicitly calculate the losses. 
1.1.2. Epidemic models. We use the network-based SIS model, also known as the contact process, to model the spread of the considered cyber threat. This continuous time Markov chain has been extensively analyzed. A key topic in the analysis is the long-term behavior of the system as a function of the model parameter $\tau$ - the ratio of the infection to the curing rate. For networks of infinite size, both survival and extinction of the considered threat can occur. For example, in the network $\mathbb{Z}^{k}$, there exists a critical value $\tau_{c}$ such that for $\tau \leq \tau_{c}$, the infection dies out, while for $\tau>\tau_{c}$, the infection survives (cf. Liggett (1985) for the case $k=1$, and Bezuidenhout and Grimmett (1990) and Liggett (1999), Part I, Section 2, for $k \geq 1$ ). For networks of finite size, however, the infection will almost surely die out in finite time. This is due to the presence of the absorbing healthy state in the Markov chain. Still, there is a different kind of threshold behavior of the system: There exists a critical value for $\tau$ that determines the behavior of the expected extinction time as a function of the network size $N$. Below this critical value, the expected extinction time increases logarithmically for increasing $N$; above, it increases exponentially fast (see, e.g., Durrett and Liu (1988) for an analysis of the chain graph $\{1, \ldots, N\}$ and Mountford et al. (2016) for bounds on the expected survival time for the homogeneous tree of bounded degree). For a recent survey paper, including bounds in general graphs, we refer to Nowzari et al. (2016, Theorems 4 and 5).

In our insurance application, we are mainly interested in the moments of the Markovian spread process. These can be used to approximate expected losses and to compute insurance premiums. Since the exact calculation of these moments requires the solution of a system of $2^{N}-1$ ODEs, we use a lower order mean-field approximation in the sense of Van Mieghem et al. (2009). In contrast to the degree-based mean-field approach (Pastor-Satorras and Vespignani, 2001; Boguñá and Pastor-Satorras, 2002), which builds on average degrees, this individual-based approach captures the complete structure of the network. This enables us to analyze the influence of the network topology on the spread of the infection and on insured losses.

Van Mieghem et al. (2009) derive a first-order independent mean-field approximation called NIMFA. We add an analysis of its accuracy. In contrast to earlier papers (such as Van Mieghem and van de Bovenkamp (2015)), we provide a time-dependent accuracy criterion that is able to qualitatively capture the behavior of the approximation error over time, if the parameter $\tau$ is sufficiently small. For larger values of $\tau$, however, we observe that first-order approximations may lead to substantial errors. In this case, we propose not to use a first-, but a higher order approximation.

The fact that the mean-field approximation approach can be generalized to higher orders has already been noted previously; we refer to Cator and Van Mieghem (2012) for a second-order independent approximation with naive single split, to Mata and Ferreira (2013) for a second-order pair-approximation, and to Pastor-Satorras et al. (2015, p.19) for a recent review. The present paper provides the first explicit derivation of a general nth-order mean-field approximation, i.e., an approximation of the moments of the spread process up to order $n$. 
We show that our $n$ th-order mean-field approximation is defined by two main ingredients: a mean-field function and a splitting algorithm. Our framework comprises the previous contributions Van Mieghem et al. (2009) and Cator and Van Mieghem (2012) as special cases. It is also more general than these papers and enables us to introduce and analyze a new Hilbert approximation and splitting algorithms.

1.1.3. Marked point processes. To model the claims, we use a marked point process. A general introduction to this type of processes as well as the main theoretical results can be found in Jacod (1975), Brémaud (1981) and Last and Brandt (1995). Marked point processes have been applied in many different areas such as credit risk modeling (see, e.g., Bielecki and Rutkowski, 2004), survival analysis (see, e.g., Jacobsen, 2006, Chapter 8), and insurance loss modeling (see, e.g., Burnecki et al., 2011). In this paper, we build on the latter approach; however, our loss process is additionally coupled with the underlying spread process of the cyber infection. This captures the idea that only infected nodes may suffer losses. A similar approach is used in Giesecke and Weber (2004) and Giesecke and Weber (2006) in the context of liquidity and credit risk.

\subsection{Outline}

The paper is organized as follows. Section 2 presents our exact loss model. Section 2.1 introduces the SIS Markov chain as a model of the infection process. Section 2.2 describes the marked point process that generates the claims at infected sites. A formula for the expected aggregate losses of a reinsurer is derived. Section 3 explains different approximations. Section 3.1 presents a polynomial approximation approach to evaluate non-linear claim functions. Section 3.2 introduces mean-field approximations of the moments of the spread process. We first analyze first-order approximations and the corresponding ODE systems. Second, we define the general $n$ th-order approximation and its two key ingredients: the mean-field function and the splitting algorithm. Section 4 illustrates in numerical case studies how the suggested approach may be applied to the pricing of different insurance contracts. This allows to study the influence of the underlying network topology. Section 5 concludes. All proofs can be found in an appendix.

\section{EXACT LOSS MODEL}

We consider a cyber threat that spreads via two consecutive channels. First, a vulnerability is created by an infection in a given network of agents. More specifically, each agent (a corporation, a system of computers or a single device) is represented by a node in the network; each edge constitutes a possible transmission channel of the cyber infection. An edge could be a direct link between individual agents, or a link to a central server that stores data or supplies users with 
software updates. Second, infected agents are vulnerable to randomly occurring attacks, triggering losses of random size. For example, an infected computer may be attacked by collecting and abusing private information such as credit card or banking information, or by disturbing operations on the computer via ransomware. If agents are insured against cyber damage, insurance claims depend on two different types of stochastic processes: first, the infectious spread process of the vulnerability, and, second, the claim frequency and severity processes.

\subsection{Spread process}

The cyber network consists of $N$ agents, labeled $1, \ldots, N$. To begin with, we focus in the current paper on a simple undirected graph. The suggested model could easily be extended to directed and weighted graph structures. Such extensions could provide more realistic models of cyber networks with asymmetric infection channels of different strengths, but their analysis would be more involved, and we thus leave a detailed analysis of complex networks to future research. The undirected network is represented by a symmetric adjacency matrix $A \in\{0,1\}^{N \times N}$, with $a_{i i}=0$ for all $i$, where $a_{i j}=1$ indicates a connection between nodes $i$ and $j$ and $a_{i j}=0$ signifies that $i$ and $j$ are not directly connected.

To describe the dynamics of the first channel, the infectious spread of vulnerability to cyber events, we use the SIS model, as, for example, explained in Pastor-Satorras et al. (2015, Section V). At any point in time, each node $i$ can be in one of two states: infected or susceptible. The state of node $i$ at time $t$ is denoted by $X_{i}(t)$, where $X_{i}(t)=1$ indicates that node $i$ is infected at time $t$ and $X_{i}(t)=0$ indicates that node $i$ is susceptible to an infection. We assume that each node can be infected by its infected neighbors, but is cured independently of all other nodes in the system. We assume that each node is endowed with an independent exponential clock and changes its state when the exponential clock rings. Letting $\beta>0$ and $\delta>0$, the rates of these transitions are given as follows $(i=1,2, \ldots, N)$ :

$$
\begin{aligned}
& X_{i}: 0 \rightarrow 1 \quad \text { with rate } \beta \sum_{j=1}^{N} a_{i j} X_{j}(t) \\
& X_{i}: 1 \rightarrow 0 \text { with rate } \delta .
\end{aligned}
$$

To be precise, we will from now on work on a probability space $(\Omega, \mathcal{F}, P)$ with filtration $\mathbb{F}=\left(\mathcal{F}_{t}\right)_{t \geq 0}$ that satisfies the usual conditions, i.e., the filtration is right-continuous and $\mathcal{F}_{0}$ contains all $P$-null sets (see, e.g., Protter, 2004). The process $X$ is a Markov-process with state space $E=\{0,1\}^{N}$ with càdlàg paths and $X_{0}=x \in E$. We assume that $X$ is a Feller process with generator $G$ : 
$C(E) \rightarrow \mathbb{R}$ defined by

$G f(x)=\sum_{i=1}^{N}\left(\beta\left(1-x_{i}\right) \sum_{j=1}^{N} a_{i j} x_{j}+x_{i} \delta\right)\left(f\left(x^{i}\right)-f(x)\right), \quad x \in E, f \in C(E)$,

where $x_{j}^{i}=x_{j}$ for $i \neq j$ and $x_{i}^{i}=1-x_{i}$. The family $C(E)$ consists of all functions on $E$. For details, we refer to Liggett (1985).

The continuous-time Markov process $X$ is of pure jump-type with exponential waiting times between jumps. The dimension of the state space $E$ is large, i.e., $E$ has cardinality $2^{N}$. In Section 3.2, we derive a tractable mean-field approximation in terms of a system of ODEs that can be applied to the valuation of cyber insurance.

\subsection{Claims processes}

In our model of cyber losses and insurance, we assume that the process $X$ does not directly cause any damage. Instead, at each point in time, infected agents are vulnerable to cyber attacks, while agents who are not infected are not. For example, infected agents might constitute a botnet that enables a denial-of-service or ransomware attack.

In order to model cyber losses, we assume that the attacks are counted by a process $M:=(M(t))_{t \geq 0}$ with values in $\{0,1,2, \ldots\}$. The corresponding jump times are denoted by $\left(T_{n}\right)_{n \in \mathbb{N}}$. The size of possible losses at each site during an attack is modeled by another $N$-dimensional process $L:=(L(t))_{t>0}$, where $L(t)=\left(L_{1}(t), \ldots, L_{N}(t)\right)^{\top}$. We assume that both $M$ and $L$ are independent from the Markovian spread process $X$.

To be precise, $M$ is a non-explosive counting process adapted to the filtration $\mathbb{F}$. We suppose that $M$ has a stochastic intensity $(\lambda(t))_{t \geq 0}$, i.e., $\lambda$ is some nonnegative $\mathbb{F}$-predictable process with $M\left(t \wedge T_{n}\right)-\int_{0}^{t \wedge T_{n}} \lambda(s) d s$ is a martingale for all $n \in \mathbb{N}$ (see Brémaud, 1981, T9/p. $28 \&$ T13/p. 31). The loss process $L$ is assumed to be predictable and non-negative.

We consider a (re-)insurance contract covering cyber losses and compute the expected aggregate losses over a fixed time window $[0, T]$ with $T>0$. We suppose that for any time $t$ the insurance contract is characterized by a function $f(\cdot ; \cdot): \mathbb{R}_{+} \times \mathbb{R}_{+}^{N} \rightarrow \mathbb{R}_{+}$, where the first argument refers to time and the second argument to the loss vector generated by a cyber attack. We suppose that $f$ is jointly measurable.

We denote the Hadamard product of vectors (i.e., the multiplication of the components) by $\circ$. At time $t$, the insurance contract covers $f(t ; L(t) \circ X(t))$, if a loss event occurs at time $t$. Neglecting interest rates or considering discounted quantities, the expected aggregate losses of the contract over the time window 
$[0, T]$ are thus given by

$$
\begin{aligned}
\mathbb{E}\left[\int_{0}^{T} f(t ; L(t) \circ X(t)) d M(t)\right] & =\mathbb{E}\left[\int_{0}^{T} f(t ; L(t) \circ X(t-)) d M(t)\right] \\
=\mathbb{E}\left[\int_{0}^{T} f(t ; L(t) \circ X(t-)) \lambda(t) d t\right] & =\mathbb{E}\left[\int_{0}^{T} f(t ; L(t) \circ X(t)) \lambda(t) d t\right] .
\end{aligned}
$$

The first equality is due to the fact that $X$ and $M$ are independent and never jump at the same time with probability 1 . The second equality follows from the predictability of the integrand according to D7/p. 27 in Brémaud (1981). The third equality holds, since the paths of $X$ possess at most countably many jumps on $[0, T]$ and constitute a Lebesgue null set for each path.

The simplest contract $f$ is a proportional insurance, i.e., $f(t ; z)=\sum_{i=1}^{N} \alpha_{i} z_{i}$. In this case,

$$
\mathbb{E}\left[\int_{0}^{T} f(t ; L(t) \circ X(t)) d M(t)\right]=\int_{0}^{T} \sum_{i=1}^{N} \alpha_{i} \cdot \mathbb{E}\left[X_{i}(t)\right] \cdot \mathbb{E}\left[L_{i}(t) \lambda(t)\right] d t,
$$

where the factorization follows from the independence of $X$ and $(M, \lambda, L)$.

For a linear claim function, the computation of the expected losses of the insurance contract does not require full knowledge of the dynamics of the spread process $X$, but only of its expectation, i.e., the first moment. If $f$ is non-linear, but continuous, a polynomial approximation can be used in order to evaluate equation (2). This requires knowledge of the evolution of all moments of $X$ up to the degree that is desired for the evaluation. This will be explained in Section 3.1.

Due to Kolmogorov's equations, using the fact that the components of $X$ are idempotent and commutative, the moments of $X$ are described by a finite system of ODEs consisting of $\sum_{i=1}^{N}\left(\begin{array}{c}N \\ i\end{array}\right)=2^{N}-1$ equations. For large $N$, this coupled system of equations of first order becomes intractable. ${ }^{1}$ If the order of the polynomial approximation is less $N$, say $n$, only moments of $X$ up to order $n$ are needed. However, the ODEs for these moments depend on higher order moments, i.e., the desired system of equations is not closed. We address this problem in Section 3.2 by constructing a mean-field approximation of the dynamics of the desired moments that significantly reduces the dimension of the system of ODEs.

\section{APPROXIMATIONS}

\subsection{Polynomial approximation of non-linear claim functions}

In this section, we discuss a polynomial approximation of the claim function $f$ that facilitates the computation of expected insurance losses (2) in the non-linear 
case. In order to simplify the mathematical analysis, we assume in this paper that $f$ does not depend on $t$. We do, however, stress that our analysis can be extended to the time-dependent case at the expense of constructing approximations that are sufficiently regular in time. This requires a more complicated notation. The basic idea of the polynomial approximation is that any continuous function $f$ can be uniformly approximated by polynomials on any compact set according to the Stone-Weierstraß theorem. We make the following assumptions.

\section{Assumption 3.1.}

i. The function $f: \mathbb{R}_{+}^{N} \rightarrow \mathbb{R}_{+}$is decomposable, i.e., one can write

$$
f\left(x_{1}, \ldots, x_{N}\right)=g\left(\Lambda\left(x_{1}, \ldots, x_{N}\right)\right),
$$

where $\Lambda: \mathbb{R}_{+}^{N} \rightarrow \mathbb{R}_{+}$is a linear and increasing aggregation function and $g: \mathbb{R}_{+} \rightarrow \mathbb{R}_{+}$is continuous and increasing.

ii. The function $g$ is bounded on $\left[0,\|\Lambda(L)\|_{\infty}\right)$, where $\|\cdot\|_{\infty}$ denotes the $L^{\infty}$ norm.

\section{Example 3.2.}

i. A first example is a catastrophe excess of loss per risk (Cat XL) contract that covers cyber attacks with priority $a \geq 0$ and limit $b-a>0$. In this case, insurance losses are described by

$$
f^{\text {Cat-XL }}\left(x_{1}, \ldots, x_{N}\right)=g^{\text {Cat-XL }}\left(\Lambda^{\Sigma}\left(x_{1}, \ldots, x_{N}\right)\right)
$$

with $\Lambda^{\Sigma}\left(x_{1}, \ldots, x_{N}\right)=\sum_{i=1}^{N} x_{i}$, and $g^{\mathrm{Cat}-\mathrm{XL}}(y)=(y-a)^{+}-(y-b)^{+}$. This shows that $f^{\mathrm{Cat}-\mathrm{XL}}$ satisfies Assumption 3.1.

ii. Another example is an excess of loss per risk contract (XL) that covers all individual cyber losses. This is described by

$$
f^{\mathrm{XL}}\left(x_{1}, \ldots, x_{N}\right):=\sum_{i=1}^{N}\left(\left(x_{i}-a_{i}\right)^{+}-\left(x_{i}-b_{i}\right)^{+}\right),
$$

where $a_{i} \geq 0$ is the priority of risk $i$ and $b_{i}-a_{i}>0$ its corresponding cover. This function is neither linear nor does it satisfy Assumption 3.1. However, with a small trick it fits into both frameworks. To this end, observe that

$$
f^{\mathrm{XL}}(L(t) \circ X(t))=\sum_{i=1}^{N}\left(\left(L_{i}(t) X_{i}(t)-a_{i}\right)^{+}-\left(L_{i}(t) X_{i}(t)-b_{i}\right)^{+}\right) .
$$

Since $X_{i}(t) \in\{0,1\}$, this function can be rewritten in the following form:

$$
f^{\mathrm{XL}}(L(t) \circ X(t))=\sum_{i=1}^{N} \hat{L}_{i}(t) X_{i}(t)=: \hat{f}^{\mathrm{XL}}(\hat{L}(t) \circ X(t)),
$$


where $\hat{L}_{i}(t)=\left(L_{i}(t)-a_{i}\right)^{+}-\left(L_{i}(t)-b_{i}\right)^{+}$for $i=1, \ldots, N$. Then,

$$
\hat{f}^{\mathrm{XL}}\left(x_{1}, \ldots, x_{N}\right)=\sum_{i=1}^{N} x_{i} .
$$

For the modified loss size process $\hat{L}$, the function $\hat{f}^{\mathrm{XL}}$ describes a proportional reinsurance. Assumption 3.1 is satisfied with $\Lambda=\hat{f}^{\mathrm{XL}}$ and $g(\lambda)=\lambda$ for $\lambda \geq 0$.

Remark 3.3. The application of the Stone-Weierstraß theorem does not rely on the existence of a decomposition of $f$ as required in Assumption 3.1. The decomposition guarantees that we may work with polynomial approximations in one dimension. In this case, simple algorithms for the construction of the unique best approximation are available. In contrast, due to the theorem of Mairhuber-Curtis, finitedimensional subspaces of the space of continuous functions on a multi-dimensional compact set are not Haar spaces, implying that best approximations are not always unique.

If Assumption 3.1 is satisfied, a polynomial approximation can be constructed as follows.

\section{Approximation 3.4.}

$i$. Choose a bound $\epsilon>0$ and the desired degree of the polynomial approximation $d \in \mathbb{N}$.

ii. Determine a constant $u \in \mathbb{R}_{+}$such that the probability that an aggregated loss (under a total infection) exceeds $u$ is bounded from above by $\epsilon$, i.e.,

$$
P(\Lambda(L)>u) \leq \epsilon .
$$

iii. Find the best uniform approximation $p_{d}(x):=\sum_{\ell=0}^{d} a_{\ell} x^{\ell}\left(a_{0}, a_{1}, \ldots, a_{d} \in\right.$ $\mathbb{R})$ of the function $g$ on the compact interval $[0, u]$ in the space of polynomials up to degree $d$. We denote the resulting approximation error by

$$
\max _{x \in[0, u]}\left|g(x)-p_{d}(x)\right|=\left\|g-p_{d}\right\|_{\infty,[0, u]}=: e_{d}(g) .
$$

iv. The dth degree polynomial approximation of $f(L \circ X)$ is given by

$$
\bar{f}_{d}(L \circ X):= \begin{cases}p_{d}(\Lambda(L \circ X)), & \text { if } \Lambda(L) \leq u, \\ 0, & \text { if } \Lambda(L)>u .\end{cases}
$$

Remark 3.5. Due to Assumption 3.1, there exists a real number $m$ s.t.

$$
|f(L \circ X)|=|g(\Lambda(L \circ X))| \leq|g(\Lambda(L))| \leq m
$$

for all possible realizations of the random variable $L$. The $L^{1}$-norm of the approximation error for $\bar{f}_{d}(L \circ X)$ is bounded from above by $e_{d}(g)+m \cdot \epsilon$. This can easily 
be verified. Letting $Z=L \circ X$, we obtain that

$$
\begin{aligned}
\left\|f(Z)-\bar{f}_{d}(Z)\right\|_{L_{1}}= & \|\left[f(Z)-p_{d}(\Lambda(Z))\right] \cdot \mathbb{1}_{[0, u]}(\Lambda(L)) \\
& +f(Z) \cdot \mathbb{1}_{(u, \infty)}(\Lambda(L)) \|_{L_{1}} \\
\leq & \mathbb{E}\left[\left|g(\Lambda(Z))-p_{d}(\Lambda(Z))\right| \cdot \mathbb{1}_{[0, u]}(\Lambda(L))\right] \\
& +\mathbb{E}\left[|f(Z)| \cdot \mathbb{1}_{(u, \infty)}(\Lambda(L))\right] \\
& \stackrel{(\circ)}{\leq}\left\|g-p_{d}\right\|_{\infty,[0, u]} \cdot P(\Lambda(L) \leq u)+m \cdot P(\Lambda(L)>u) \\
\leq & e_{d}(g)+m \cdot \epsilon,
\end{aligned}
$$

observing in step (o) that $0 \leq \Lambda(Z) \leq \Lambda(L)$.

Finally, consider an insurance contract $f=g(\Lambda)$ with $\Lambda\left(x_{1}, \ldots, x_{N}\right)=$ $\sum_{i=1}^{N} b_{i} x_{i}, b_{1}, b_{2}, \ldots, b_{N} \geq 0$, and polynomial approximation $p_{d}(x):=$ $\sum_{\ell=0}^{d} a_{\ell} x^{\ell}$. In this case,

$$
\mathbb{E}\left[\int_{0}^{T} \bar{f}_{d}(L(t) \circ X(t)) d M(t)\right]=\int_{0}^{T} \mathbb{E}\left[\bar{f}_{d}(L(t) \circ X(t)) \cdot \lambda(t)\right] d t .
$$

Set $\mathcal{G}:=\sigma\{L(t), \lambda(t): t \leq T\}$, and observe that the process $X$ is independent of $\mathcal{G}$. Thus,

$$
\mathbb{E}\left[\bar{f}_{d}(L(t) \circ X(t)) \cdot \lambda(t) \mid \mathcal{G}\right]=\lambda(t) \cdot \mathbb{E}\left[\bar{f}_{d}(L(t) \circ X(t)) \mid \mathcal{G}\right] .
$$

We evaluate the second factor. For simplicity, we suppress the dependence on $t$ in the notation.

$$
\begin{aligned}
& \mathbb{E}\left[\bar{f}_{d}(L \circ X) \mid \mathcal{G}\right]=\mathbb{E}\left[p_{d}(\Lambda(L \circ X)) \cdot \mathbb{1}_{[0, u]}(\Lambda(L)) \mid \mathcal{G}\right] \\
& =\sum_{\ell=0}^{d} a_{\ell} \cdot \mathbb{E}\left[(\Lambda(L \circ X))^{\ell} \mid \mathcal{G}\right] \cdot \mathbb{1}_{[0, u]}(\Lambda(L)) \\
& =\sum_{\ell=0}^{d} a_{\ell} \cdot \mathbb{E}\left[\left(\sum_{i=1}^{N} b_{i} L_{i} X_{i}\right)^{\ell} \mid \mathcal{G}\right] \cdot \mathbb{1}_{[0, u]}(\Lambda(L)) \\
& =\mathbb{1}_{[0, u]}(\Lambda(L)) \cdot\left[a_{0}+a_{1} \sum_{i_{1}=1}^{N} b_{i_{1}} L_{i_{1}} \mathbb{E}\left[X_{i_{1}}\right]+a_{2} \sum_{i_{1}=1}^{N} \sum_{i_{2}=1}^{N} b_{i_{1}} b_{i_{2}} L_{i_{1}} L_{i_{2}} \mathbb{E}\left[X_{i_{1}} X_{i_{2}}\right]\right. \\
& \left.\quad+\ldots+a_{d} \sum_{i_{1}=1}^{N} \sum_{i_{2}=1}^{N} \ldots \sum_{i_{d}=1}^{N} b_{i_{1}} b_{i_{2}} \cdots b_{i_{d}} \cdot L_{i_{1}} L_{i_{2}} \cdots L_{i_{d}} \cdot \mathbb{E}\left[X_{i_{1}} X_{i_{2}} \cdots X_{i_{d}}\right]\right] .
\end{aligned}
$$

Here, we used the fact that the process $L$ is $\mathcal{G}$-measurable, while $X$ is independent of $\mathcal{G}$. In the next step, we can now use the tower property on the conditional 
expectations in equation (4), i.e., $\mathbb{E}[\mathbb{E}(\cdot \mid \mathcal{G})]=\mathbb{E}(\cdot)$, to obtain the final result. Thus, in summary, for the purpose of approximating the expected insurance losses, we need to calculate

$$
\begin{gathered}
\int_{0}^{T} \mathbb{E}\left(\mathbb { 1 } _ { [ 0 , u ] } ( \Lambda ( L ) ) \cdot \lambda ( t ) \cdot \left[a_{0}+a_{1} \sum_{i_{1}=1}^{N} b_{i_{1}} L_{i_{1}} \mathbb{E}\left[X_{i_{1}}\right]+a_{2} \sum_{i_{1}=1}^{N} \sum_{i_{2}=1}^{N} b_{i_{1}} b_{i_{2}} L_{i_{1}} L_{i_{2}} \mathbb{E}\left[X_{i_{1}} X_{i_{2}}\right]\right.\right. \\
\left.\left.+\ldots+a_{d} \sum_{i_{1}=1}^{N} \sum_{i_{2}=1}^{N} \cdots \sum_{i_{d}=1}^{N} b_{i_{1}} b_{i_{2}} \cdots b_{i_{d}} \cdot L_{i_{1}} L_{i_{2}} \cdots L_{i_{d}} \cdot \mathbb{E}\left[X_{i_{1}} X_{i_{2}} \cdots X_{i_{d}}\right]\right]\right) d t
\end{gathered}
$$

The formula shows that full knowledge of the probabilistic evolution of the process $X$ is not required. Instead, an analysis of the dynamics of its moments up to order $d$ suffices to compute the sought quantity. As explained earlier, the corresponding system of ODEs is obtained from Kolmogorov's equations, but involves $2^{N}-1$ equations. A closed system for the required moments with a smaller number of equations can be obtained by a mean-field approximation. This method will be explained in the next section.

\section{Remark 3.6.}

$i$. If the network size $N$ is not too large, the polynomial approximation enables a flexible and efficient comparison of different contracts in a given network. The mixed moments of the spread process need to be computed only once. Afterwards, polynomial approximations of different contracts can be evaluated. Since the number of mixed moments up to order d grows like $N^{d}$ when the number of nodes $N$ increases, polynomial approximations are not efficient anymore for very large networks.

ii. In this paper, approximations of the joint moments of the spread process are computed from an ODE system. The methodology is explained in the next section. Alternatively, joint moments could be computed from Monte Carlo simulations of the spread process. A comparison of the advantages and disadvantages of these competing approaches is beyond the scope of the current paper and constitutes an interesting topic for future research.

\subsection{Mean-field approximation of moments}

In order to evaluate formula (5), we need to compute the moments $\mathbb{E}\left[X_{i_{1}}(t)\right]$, $\mathbb{E}\left[X_{i_{1}}(t) X_{i_{2}}(t)\right], \ldots, \mathbb{E}\left[X_{i_{1}}(t) X_{i_{2}}(t) \cdots X_{i_{n}}(t)\right], i_{1}, \ldots, i_{n} \in\{1, \ldots, N\}$, for the desired $n \leq N$. We construct an approximation of these moments and call the parameter $n$ its order. For this purpose, we denote by $z_{i_{1} i_{2} \ldots i_{k}}^{(n)}$ the $n$ th-order approximation of the moment $\mathbb{E}\left[X_{i_{1}} X_{i_{2}} \cdots X_{i_{k}}\right]$ for $k \leq n$. Observe that both $z_{i_{1} i_{2} \ldots i_{k}}^{(n)}$ and $\mathbb{E}\left[X_{i_{1}} X_{i_{2}} \cdots X_{i_{k}}\right]$ are functions of $t$. In order to simplify the notation, we will sometimes drop the variable $t$ in our notation. Since the variables 
$X_{i}, i=1,2, \ldots, n$, are commutative and idempotent, we may assume that the indices $i_{1}, \ldots, i_{k}$ are pairwise different and ordered, i.e., $i_{1}<i_{2}<\ldots<i_{k}$. Alternatively, the $n$ th-order approximation of all moments of $X$ up to order $n$ can simply be enumerated by index sets $I \subseteq\{1,2, \ldots, N\}$ with cardinality $|I| \leq n$. This will be the convention that we choose. As a final result of our construction, we obtain the $n$ th-order approximation of all moments of $X$ up to order $n$, i.e.,

$$
\left(z_{I}^{(n)}\right)_{I \subseteq\{1,2, \ldots, N\},|I| \leq n} .
$$

We begin with a detailed description of the first-order approximation, explaining two strategies for its construction. This approach is later generalized to $n$ thorder approximations for $n>1$.

3.2.1. First order. We derive the first-order mean-field approximation in detail and analyze its accuracy.

Derivation of the approximations: The transition rates (1) describe the infinitesimal dynamics of $X_{i}(t)$

$$
\mathbb{E}\left[X_{i}(t+\Delta t)-X_{i}(t) \mid \mathcal{F}_{t}\right]=\left(\left(1-X_{i}(t)\right) \beta \sum_{j=1}^{N} a_{i j} X_{j}(t)-\delta X_{i}(t)\right) \Delta t+o(\Delta t) .
$$

A susceptible node is infected by its neighbors at rate $\beta$; an infected node is cured at rate $\delta$, independently of the state of the others. Intuitively, dividing by $\Delta t$, taking the expectation on both sides and letting $\Delta t \rightarrow 0$, we obtain the following exact expression for the derivative of the probability $\mathbb{E}\left[X_{i}(t)\right]=$ $P\left(X_{i}(t)=1\right)$ :

$$
\frac{d \mathbb{E}\left[X_{i}(t)\right]}{d t}=-\delta \mathbb{E}\left[X_{i}(t)\right]+\beta \sum_{j=1}^{N} a_{i j} \mathbb{E}\left[X_{j}(t)\right]-\beta \sum_{j=1}^{N} a_{i j} \mathbb{E}\left[X_{i}(t) X_{j}(t)\right],
$$

for $i=1, \ldots, N$. More precisely, these equations are a consequence of Kolmogorov's forward equation.

The occurrence of the mixed terms $\mathbb{E}\left[X_{i}(t) X_{j}(t)\right]$ for $i \neq j$ signifies that the dynamics of the first-order moments depend on higher order moments. The system of ODEs (7) is not closed. An exact solution requires in addition the dynamics of the second-order moments, third-order moments, etc. Instead of increasing the number of equations, the aim of the first-order mean-field approximation is to keep the number of equations fixed, but to pay the price of obtaining an approximate in lieu of an exact solution.

We choose a suitable function $F:[0,1] \rightarrow[0,1]$ and split the mixed terms as

$$
\mathbb{E}\left[X_{i}(t) X_{j}(t)\right] \approx F\left(\mathbb{E}\left[X_{i}(t)\right]\right) \cdot F\left(\mathbb{E}\left[X_{j}(t)\right]\right) .
$$


This leads to the following approximation:

$$
\begin{aligned}
\frac{d \mathbb{E}\left[X_{i}(t)\right]}{d t} \approx & -\delta \mathbb{E}\left[X_{i}(t)\right]+\beta \sum_{j=1}^{N} a_{i j} \mathbb{E}\left[X_{j}(t)\right] \\
& -\beta \sum_{j=1}^{N} a_{i j} F\left(\mathbb{E}\left[X_{i}(t)\right]\right) \cdot F\left(\mathbb{E}\left[X_{j}(t)\right]\right) .
\end{aligned}
$$

We denote by $z_{i}^{(1)}(t)$ the corresponding approximation of $\mathbb{E}\left[X_{i}(t)\right]$ and arrive at the following system of ODEs:

$$
\begin{aligned}
\frac{d z_{i}^{(1)}(t)}{d t}= & -\delta z_{i}^{(1)}(t)+\beta \sum_{j=1}^{N} a_{i j} z_{i}^{(1)}(t) \\
& -\beta \sum_{j=1}^{N} a_{i j} F\left(z_{i}^{(1)}(t)\right) \cdot F\left(z_{j}^{(1)}(t)\right) \quad(i=1, \ldots, N) .
\end{aligned}
$$

This is the first-order mean-field approximation corresponding to the mean-field function $F$.

Next, we consider two examples of mean-field functions and the resulting first-order approximations $z_{i}^{(1)}(t)$ in more detail.

i. If we choose a mean-field function $F_{1}(x)=x$, we obtain the first-order independent approximation, also known as the " $N$-intertwined mean-field approximation (NIMFA)". This approximation is discussed in detail by Van Mieghem et al. (2009). The approximation factorizes the second-order moments as if components were independent

$$
\mathbb{E}\left[X_{i}(t) X_{j}(t)\right] \approx F_{1}\left(\mathbb{E}\left[X_{i}(t)\right]\right) \cdot F_{1}\left(\mathbb{E}\left[X_{j}(t)\right]\right)=\mathbb{E}\left[X_{i}(t)\right] \mathbb{E}\left[X_{j}(t)\right] .
$$

Since $\mathbb{E}\left[X_{i}(t) X_{j}(t)\right]=\mathbb{E}\left[X_{i}(t)\right] \mathbb{E}\left[X_{j}(t)\right]+\operatorname{Cov}\left(X_{i}(t), X_{j}(t)\right)$ with $\operatorname{Cov}\left(X_{i}(t), X_{j}(t)\right) \geq 0$, as shown in Cator and Van Mieghem (2014), equation (8) leads to an upper bound

$$
\frac{d \mathbb{E}\left[X_{i}(t)\right]}{d t} \leq-\delta \mathbb{E}\left[X_{i}(t)\right]+\beta \sum_{j=1}^{N} a_{i j} \mathbb{E}\left[X_{j}(t)\right]-\beta \sum_{j=1}^{N} a_{i j} \mathbb{E}\left[X_{i}(t)\right] \mathbb{E}\left[X_{j}(t)\right] .
$$

The upper bound mean-field approximation $v_{i}(t):=z_{i}^{(1)}(t)$ is characterized by the ODEs

$$
\frac{d v_{i}(t)}{d t}=-\delta v_{i}(t)+\beta \sum_{j=1}^{N} a_{i j} v_{j}(t)-\beta \sum_{j=1}^{N} a_{i j} v_{i}(t) v_{j}(t), \quad(i=1, \ldots, N) .
$$


Setting $V:=\left(v_{1}, v_{2}, \ldots, v_{N}\right)^{\top}$, we may rewrite the system in matrix notation

$$
\frac{d}{d t} V=(\beta A-\delta \mathbb{I}) V-\beta \operatorname{diag}(V) A V,
$$

where $\operatorname{diag}(V)$ denotes the diagonal matrix with entries $v_{1}, v_{2}, \ldots, v_{N}$ and $\mathbb{I} \in \mathbb{R}^{N \times N}$ denotes the identity matrix.

ii. A mean-field function $F_{2}(x):=\sqrt{x}$ leads to a new type of mean-field approximation. We call this the first-order Hilbert approximation that is motivated by the following observations. The space of square-integrable random variables $\mathcal{L}^{2}$, equipped with the scalar product $\langle R, S\rangle:=\mathbb{E}[R \cdot S]$ for $R, S \in \mathcal{L}^{2}$, forms a Hilbert space. The induced norm is denoted by $\|R\|:=\sqrt{\langle R, R\rangle}=\sqrt{\mathbb{E}\left[R^{2}\right]}$. The scalar product in Hilbert spaces defines the angle $\phi$ between vectors

$$
\langle R, S\rangle=\|R\| \cdot\|S\| \cdot \cos \phi .
$$

The idea is now to use $\|R\| \cdot\|S\|$ as an approximation for $\langle R, S\rangle$. The term $\|R\| \cdot\|S\|$ is never smaller than $\langle R, S\rangle$. The approximation is good, if the angle of $R$ and $S$ is close to $0^{\circ}$. Applying the approximation to equations (7), we obtain

$$
\begin{aligned}
\frac{d \mathbb{E}\left[X_{i}(t)\right]}{d t} & \geq-\delta \mathbb{E}\left[X_{i}(t)\right]+\beta \sum_{k=1}^{N} a_{i k} \mathbb{E}\left[X_{k}(t)\right] \\
& -\beta \sum_{k=1}^{N} a_{i k} \sqrt{\mathbb{E}\left[X_{i}(t)\right]} \sqrt{\mathbb{E}\left[X_{k}(t)\right]}, \quad i=1,2, \ldots, N .
\end{aligned}
$$

Setting $w_{i}(t):=z_{i}^{(1)}(t)$, this leads to the following mean-field approximation:

$$
\begin{aligned}
\frac{d w_{i}(t)}{d t} & =-\delta w_{i}(t)+\beta \sum_{k=1}^{N} a_{i k} w_{k}(t) \\
& -\beta \sum_{k=1}^{N} a_{i k} \sqrt{w_{i}(t)} \sqrt{w_{k}(t)}, \quad(i=1, \ldots, N) .
\end{aligned}
$$

Setting $W=\left(w_{1}, \ldots, w_{N}\right)^{\top}$ and $\sqrt{W}=\left(\sqrt{w_{1}}, \ldots, \sqrt{w_{N}}\right)^{\top}$, we may rewrite the system in matrix notation

$$
\frac{d W}{d t}=(\beta A-\delta \mathbb{I}) W-\beta \operatorname{diag}(\sqrt{W}) A \sqrt{W} .
$$




$$
A:=\left(\begin{array}{lllllll}
0 & 1 & 0 & 0 & 1 & 1 & 1 \\
1 & 0 & 1 & 1 & 0 & 0 & 1 \\
0 & 1 & 0 & 1 & 1 & 1 & 0 \\
0 & 1 & 1 & 0 & 1 & 0 & 1 \\
1 & 0 & 1 & 1 & 0 & 1 & 0 \\
1 & 0 & 1 & 0 & 1 & 0 & 1 \\
1 & 1 & 0 & 1 & 0 & 1 & 0
\end{array}\right)
$$

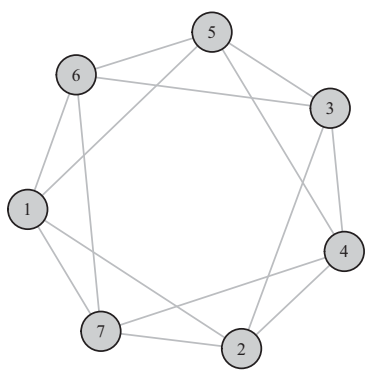

FIGURE 1: A regular network with $N=7$ nodes and degree $D=4$.

Properties of ODE systems: The following theorem summarizes key properties of the mean-field approximations. Its proof can be found in Appendix A. Let $\mathbf{0}=(0,0, \ldots, 0)^{\top} \in \mathbb{R}^{N}$ and $\mathbf{1}=(1,1, \ldots, 1)^{\top} \in \mathbb{R}^{N}$ denote the all-zero and the all-one vector, respectively.

\section{Theorem 3.7.}

$i$. Existence. For any choice of the parameters $\delta$ and $\beta$, there exist global solutions of the ODE systems (11) and (13) with arbitrary non-negative initial conditions.

ii. Uniqueness. The ODE system (11) possesses a unique solution in $C\left([0, \infty) ; \mathbb{R}^{N}\right)$.

iii. Sandwich property. Let $W(t)$ be a solution of (13) and $V(t)$ the solution to (11). For every initial condition $V(0)=W(0)=X(0)=v_{0} \in[0,1]^{N}$, it holds that

$$
\mathbf{0} \leq W(t) \leq \mathbb{E}[X(t)] \leq V(t) \leq \mathbf{1},
$$

for all $t \geq 0$, where the inequalities are interpreted componentwise.

$i v$. Stability. Let $\hat{\mu}$ be the spectral radius of $A$ and set $\tau_{c}^{(1)}:=1 / \hat{\mu}$, a constant that depends on the underlying network. Define $\tau=\beta / \delta$. If $\tau<\tau_{c}^{(1)}$, then the zero solutions $V \equiv 0$ and $W \equiv 0$ are exponentially stable solutions of (11) and (13), i.e., there exist constants $\alpha, \epsilon, C>0$ such that for any solution $z$ of (11) and (13), respectively, and all $t \geq 0$

$$
|z(t)| \leq C e^{-\alpha t|z(0)|},
$$

if $|z(0)| \leq \epsilon$.

We illustrate the two suggested approximation methodologies in the context of a small cyber network.

Example 3.8. We consider a system of $N=7$ agents that are connected in a cyber network as described in Figure 1.

The network is regular, i.e., each node is connected to exactly $D$ other nodes. We choose $D=4, \beta=0.5$ and $\delta=2.01$. Then, $\tau=\beta / \delta=0.2488<$ $1 / D=\tau_{c}^{(1)}$, thus $V \equiv 0$ and $W \equiv 0$ are exponentially stable according to 


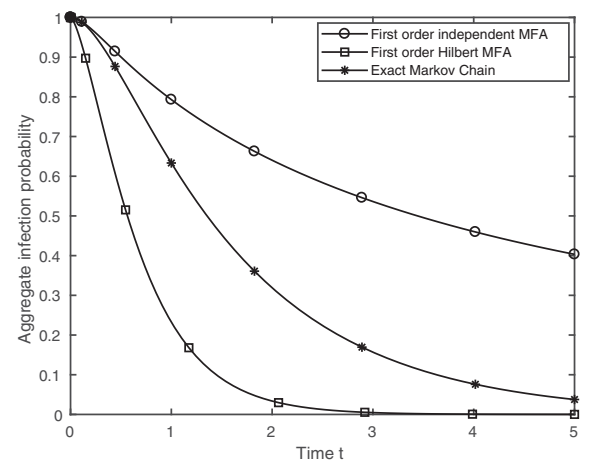

(A)

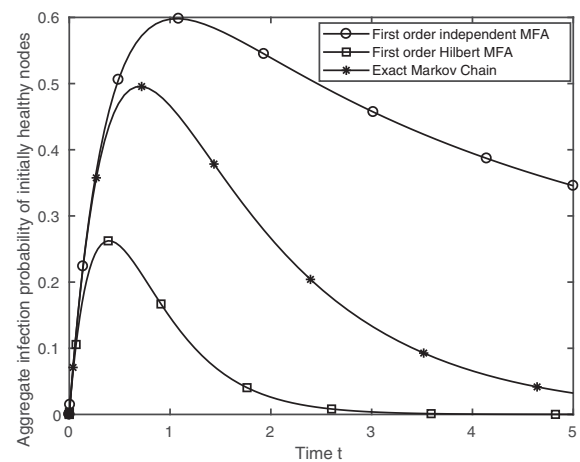

(B)

FIGURE 2: Comparison of the aggregate infection probability of all nodes (A) and of initially healthy nodes (B) for the network described in Figure 1.

Theorem 3.7 iv. We assume that initially only node 1 is infected, i.e., $V(0)=$ $W(0)=(1,0,0,0,0,0,0)^{\top}$. Figure 2 shows the aggregate infection probabilities for all nodes $(A)$, i.e., $\sum_{j=1}^{N} \mathbb{E}\left[X_{j}(t)\right]$, and respectively for all initially healthy nodes $(B)$, here: $\sum_{j=2}^{N} \mathbb{E}\left[X_{j}(t)\right]$, for the original Markov process together with the corresponding approximations. As explained in Theorem 3.7 iii., these provide lower and upper bounds. For both approximations, the error is quite substantial. As we will see in the following sections, this can be improved by higher order approximations.

Accuracy criterion: We derive a time-dependent estimate of the accuracy of the first-order independent mean-field approximation. We focus on the correlation matrix as a rough measure for the difference between the exact and approximate dynamics of the $\mathbb{E}\left[X_{i}(t)\right]$.

The first step is to rewrite (7) as

$$
\frac{d}{d t} \mathbb{E}\left[X_{i}(t)\right]=-\delta \mathbb{E}\left[X_{i}(t)\right]+\beta\left(1-\mathbb{E}\left[X_{i}(t)\right]\right) \sum_{k=1}^{N} a_{k i} \mathbb{E}\left[X_{k}(t)\right]-\beta R_{i}(t),
$$

with error term

$$
R_{i}(t):=\sum_{k=1}^{N} a_{k i} \operatorname{Cov}\left(X_{i}(t), X_{k}(t)\right)
$$

In Van Mieghem and van de Bovenkamp (2015), the authors consider $R_{i}$ as a measure for the accuracy of the approximation. They investigate numerical examples and analytical criteria to assess the smallness of $R_{i}$ in different situations. It is clear that in the independent case, yielding $R_{i}(t) \equiv 0$ for all $i$, the exact dynamics and the approximation are identical. This is the reason for our choice of the term independent approximation. Our result phrases the accuracy of the approximation as a pointwise inequality in the time variable $t$. 
Theorem 3.9. Let $y_{i}(t):=\mathbb{E}\left[X_{i}(t)\right]-v_{i}(t)$ and denote by $\hat{\mu}$ the largest eigenvalue of the adjacency matrix $A$. Then, for any $t \geq 0$, we have

$$
\|y(t)\|^{2} \leq e^{(-2 \delta+4 \beta \hat{\mu}+\beta) t} \beta \int_{0}^{t}\|R(s)\|^{2} d s,
$$

where $y(t)=\left(y_{1}(t), \ldots, y_{N}(t)\right)$ and $R(t)=\left(R_{1}(t), \ldots, R_{N}(t)\right)$.

The proof of Theorem 3.9 is given in Appendix A. To apply this result, we approximate the norm of the residual term $R(t)$ by

$$
\begin{aligned}
\|R(t)\|^{2} & =\sum_{i=1}^{N}\left(\sum_{k=1}^{N} a_{k i} \operatorname{Cov}\left(X_{i}(t), X_{k}(t)\right)\right)^{2} \leq \frac{1}{16} \sum_{i=1}^{N}\left(\sum_{k=1}^{N} a_{k i}\right)^{2} \\
& \leq \frac{N}{16} \sum_{i=1}^{N} \sum_{k=1}^{N} a_{i k}=\frac{N \ell}{8},
\end{aligned}
$$

using Cauchy's inequality and denoting by $\ell$ the total number of links within the network. Hence, we find the following upper bound for the mean-field approximation error, expressed solely through known characteristics of the given network:

$$
\|y(t)\|^{2} \leq \frac{\beta N \ell}{8} \cdot t e^{(-2 \delta+4 \beta \hat{\mu}+\beta) t} .
$$

In the case that $-2 \delta+4 \beta \hat{\mu}+\beta<0$, i.e., for small $\tau=\beta / \delta<\frac{1}{2 \hat{\mu}+1 / 2}$, the approximation error becomes small quite quickly. This corresponds to a high curing rate $\delta$ in comparison to the infection rate $\beta$. Observe that the stated exponential decay is a useful result for $t$ sufficiently large. Since $N \ell$ can be very large, equation (14) does not provide substantial information regarding the approximation error for small $t$.

Example 3.10. We consider the network in Figure 1 with fixed infection rate $\beta=0.5$, but different curing rate $\delta$. The initial state is $V(0)=(1,0,0,0,0,0,0)^{\top}$, i.e., only node 1 is infected at time zero. Figure $3(A)$ depicts the exact approximation error $\|y(t)\| .^{2}$ The upper bound from equation (14) is shown in Figure $3(B)$. Although quantitatively strongly different, both figures $(A)$ and $(B)$ exhibit the exponential decay that we proved for the upper bound.

The observation that the approximation error decreases for an increasing curing $\operatorname{rate}^{2} \delta$ is in line with the heuristic arguments of Van Mieghem et al. (2009) who describe a good performance of the first-order mean-field approximation for small $\tau$. For intermediate values of $\tau$, however, these authors expect significantly larger first-order approximation errors. In addition, if $-2 \delta+4 \beta \hat{\mu}+\beta \geq 0$, the upper bounds from Theorem 3 and equation (14) are not useful anymore, since they grow exponentially in time $t$. These facts motivate the higher order approximations that we consider later. 


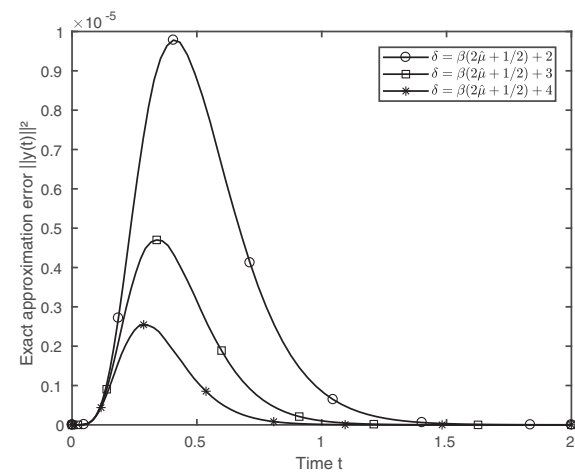

(A) Exact approximation error $\|y(t)\|^{2}$

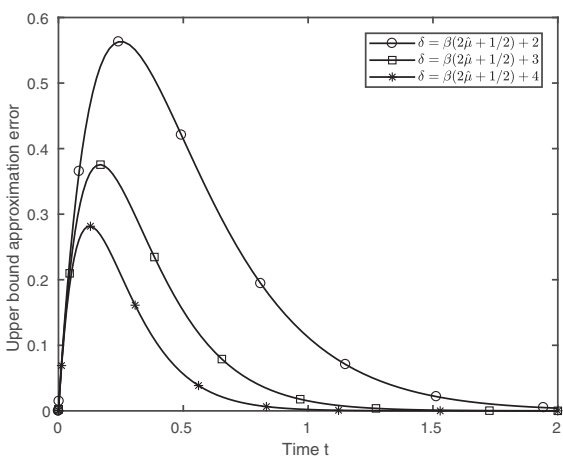

(B) Upper error bound from (14)

FIGURE 3: Exact approximation error and upper bound for different values of the curing rate $\delta$ and fixed $\beta=1 / 2$ in the regular network $A$ (given in Figure 1) with initial state $V(0)=(1,0,0,0,0,0,0)^{\top}$.

Remark 3.11. From the point of view of an insurance company, cyber risk is manageable for sufficiently small $\tau=\frac{\beta}{\delta}$. The exact condition $-2 \delta+4 \beta \hat{\mu}+\beta<0 \Leftrightarrow$ $\tau<\frac{1}{2 \hat{\mu}+1 / 2}$ depends on $\hat{\mu}$, i.e., on a characteristic of the underlying network. In this situation, the infection quickly dies out, and the first-order mean-field approximation provides an upper bound with known bound for the approximation error. It might, thus, be used for pricing insurance contracts. For small $t$, however, the error bound is large and in many cases not very precise, although the approximation might be quite reasonable.

\subsection{2. $n$th Order.}

In this section, we construct a general $n$ th-order mean-field approximation that has the following two benefits:

- The approximation error of the first-order mean-field approximations may be quite large for certain parameter choices. The $n$ th-order approximation provides improved approximations for all moments of order $k \leq n$. In particular, it yields improved estimates of single infection probabilities $z_{i}^{(n)}$ for $i=1, \ldots, N$.

- On the basis of the $n$ th-order mean-field approximation, expected insurance losses of non-linear claims can be computed as described in Section 3.1.

Construction: Fix the order $n \leq N$. By $I \subseteq\{1,2, \ldots, N\}$ we denote a set of indices. We define the product $X_{I}:=\Pi_{i \in I} X_{i}$. Since the components of $X$ are commutative and idempotent, we may neglect the order of the indices or powers of its components.

The dynamics of all moments are described by coupled ODEs due to Kolmogorov's forward equations. This system of equations is replaced by a smaller 
system of equations that involves only approximations of moments up to order $n$ :

i. Construct an approximation for the dynamics of the $n$ th-order moments $\mathbb{E}\left[X_{i_{1}} \cdots X_{i_{n}}\right]$ in terms of moments up to order $\mathrm{n}$.

ii. Use the exact relations of the dynamics of $\mathbb{E}\left[X_{i_{1}} \cdots X_{i_{k}}\right]$ for $1 \leq k \leq$ $n-1$ and the approximation of the $n$ th-order moments to estimate the lower order moments.

The details are as follows.

Step i: Approximative dynamics of $\mathbb{E}\left[X_{I}\right]$ for $|I|=n$. Kolmogorov's forward equation implies that

$$
\begin{aligned}
\frac{d}{d t} \mathbb{E} & {\left[X_{i_{1}} X_{i_{2}} \cdots X_{i_{n}}\right] } \\
= & \mathbb{E}\left[\sum_{l=1}^{n} X_{i_{1}} \cdots X_{i_{l-1}}\left(-\delta X_{i_{l}}+\beta\left(1-X_{i_{l}}\right) \sum_{j_{l}=1}^{N} a_{i_{l} j_{l}} X_{j_{l}}\right) X_{i_{l+1}} \cdots X_{i_{n}}\right] \\
= & -n \delta \mathbb{E}\left[X_{i_{1}} \cdots X_{i_{n}}\right]+\beta \mathbb{E}\left[\sum_{l=1}^{n} \sum_{j_{l}=1}^{N} a_{i_{l} j_{l}} X_{i_{1}} \cdots X_{i_{l-1}} X_{j_{l}} X_{i_{l+1}} \cdots X_{i_{n}}\right] \\
& -\beta \mathbb{E}\left[\sum_{l=1}^{n} \sum_{j_{l}=1}^{N} a_{i_{l} j_{l}} X_{i_{1}} \cdots X_{i_{n}} \cdot X_{j_{l}}\right] .
\end{aligned}
$$

Rewriting this, we have the exact expression

$$
\frac{d}{d t} \mathbb{E}\left[X_{I}\right]=-n \delta \mathbb{E}\left[X_{I}\right]+\beta \sum_{i \in I} \sum_{j=1}^{N} a_{i j} \mathbb{E}\left[X_{I \backslash\{i\} \cup\{j\}}\right]-\beta \sum_{i \in I} \sum_{j=1}^{N} a_{i j} \mathbb{E}\left[X_{I \cup\{j\}}\right] .
$$

The expression $\mathbb{E}\left[X_{I \backslash\{i\} \cup\{j]}\right]$ contains at most $n$ factors of $X$ : If $j \notin I \backslash\{i\}$, it contains $n$ factors, and if $j \in I \backslash\{i\}$, it contains $n-1$ factors. Thus, the first sum can be expressed in terms of moments of order less than or equal to $n$.

Next, consider the term $\mathbb{E}\left[X_{I \cup\{j\}}\right]$. If $j \in I$, then $\mathbb{E}\left[X_{I \cup\{j\}}\right]=\mathbb{E}\left[X_{I}\right]$. However, if $j \notin I$, the expression contains $n+1$ different indices. This means that the dynamics of the moments up to order $n$ are not described by a closed system of ODEs. To deal with this difficulty, we extend the idea of the first-order meanfield approximation and choose the following two objects:

i. a mean-field function $F:[0,1] \rightarrow[0,1]$ and

ii. a partition scheme $\left(I_{1}, I_{2}\right)$ such that for $j \neq I$, we have $I \cup\{j\}=I_{1}(I, j) \cup$ $I_{2}(I, j)$ with $I_{1}(I, j), I_{2}(I, j) \neq \emptyset$. Since $I$ is fixed for each equation, we suppress the dependence on $I$ in our notation. 
Specific choices of these two key ingredients are addressed in the next section. When they are chosen properly, we approximate the rate of change for the $n$ thorder moments by

$$
\begin{aligned}
\frac{d}{d t} \mathbb{E}\left[X_{I}\right] \approx & -n \delta \mathbb{E}\left[X_{I}\right]+\beta \sum_{i \in I} \sum_{j=1}^{N} a_{i j} \mathbb{E}\left[X_{I \backslash\{i\} \cup\{j\}}\right]-\beta \sum_{i \in I} \sum_{j=1, j \in I}^{N} a_{i j} \mathbb{E}\left[X_{I}\right] \\
& -\beta \sum_{i \in I} \sum_{j=1, j \notin I}^{N} a_{i j} \cdot F\left(\mathbb{E}\left[X_{I_{1}(j)}\right]\right) \cdot F\left(\mathbb{E}\left[X_{I_{2}(j)}\right]\right) .
\end{aligned}
$$

This translates to the ODE

$$
\begin{aligned}
\dot{z}_{I}^{(n)}= & -n \delta z_{I}^{(n)}+\beta \sum_{i \in I} \sum_{j=1}^{N} a_{i j} z_{I \backslash\{i\}\{j\}}^{(n)}-z_{I}^{(n)} \beta \sum_{i \in I} \sum_{j=1, j \in I}^{N} a_{i j} \\
& -\beta \sum_{i \in I} \sum_{j=1, j \notin I}^{N} a_{i j} F\left(z_{I_{1}(j)}^{(n)}\right) \cdot F\left(z_{I_{2}(j)}^{(n)}\right),
\end{aligned}
$$

describing the approximative dynamics of the $n$ th-order moments.

Step ii: Exact dynamics of $\mathbb{E}\left[X_{I}\right]$ for $|I|=k<n$. For moments of order $k<n$, we write according to Kolmogorov's forward equation

$$
\frac{d}{d t} \mathbb{E}\left[X_{I}\right]=-k \delta \mathbb{E}\left[X_{I}\right]+\beta \sum_{i \in I} \sum_{j=1}^{N} a_{i j} \mathbb{E}\left[X_{I \backslash\{i\} \cup\{j\}}\right]-\beta \sum_{i \in I} \sum_{j=1}^{N} a_{i j} \mathbb{E}\left[X_{I \cup\{j\}}\right],
$$

and observe that all moments in this exact equation are of order less than or equal to $n$.

Plugging in the approximations of order $n$, we obtain the ODE system of the $n$ th-order mean-field approximation

$$
\left.\begin{array}{rl}
|I|=n: \quad \dot{z}_{I}^{(n)}= & -\left(n \delta+\beta \sum_{i \in I} \sum_{j=1, j \in I}^{N} a_{i j}\right) z_{I}^{(n)}+\beta \sum_{i \in I} \sum_{j=1}^{N} a_{i j} z_{I \backslash\{i\} \cup\{j\}}^{(n)} \\
& -\beta \sum_{i \in I} \sum_{j=1, j \notin I}^{N} a_{i j} F\left(z_{I_{1}(j)}^{(n)}\right) \cdot F\left(z_{I_{2}(j)}^{(n)}\right) \\
|I|=k<n: \quad \dot{z}_{I}^{(n)}= & -k \delta z_{I}^{(n)}+\beta \sum_{i \in I} \sum_{j=1}^{N} a_{i j} z_{I \backslash\{i\} \cup\{j\}}^{(n)}-\beta \sum_{i \in I} \sum_{j=1}^{N} a_{i j} z_{I \cup\{j\}}^{(n)},
\end{array}\right\} .
$$

The initial condition is the initial configuration of the Markovian system. 
Definition 3.12. For a mean-field function $F$ and a partition scheme, we define the $n$ th-order mean-field approximation as a solution to the ODE system (16), i.e.,

$$
\left(z_{I}^{(n)}\right)_{I \subseteq\{1,2, \ldots, N\},|I| \leq n}
$$

Remark 3.13. The structure of the ODEs in the nth-order case is analogous to the first-order case. The matrix form of the ODE system (16) as well as the generalization of the existence and uniqueness results of Theorem 3.7 to the nth-order case is discussed in Appendix $C$.

Approximation types and splitting: In this section, we introduce two specific types of higher order mean-field approximations and address the problem of optimally splitting the set $I \cup\{j\}$ for $|I|=n$ and $j \notin I$ consisting of $n+1$ different indices into the two non-empty, disjoint subsets $I_{1}(j)$ and $I_{2}(j)$. In contrast to the first-order case, this requires a specific choice that may influence the quality of the resulting mean-field approximation. As before, we consider the two mean-field functions $F_{1}(x)=x$ and $F_{2}(x)=\sqrt{x}$.

i. The choice of the mean-field function $F_{1}(x)=x$ leads to the $n$ th-order independent approximation. The approximation works as follows: For $j \notin I$,

$$
\mathbb{E}\left[X_{I \cup\{j\}}\right]=\mathbb{E}\left[X_{I_{1}(j)}\right] \cdot \mathbb{E}\left[X_{I_{2}(j)}\right]+\operatorname{Cov}\left(X_{I_{1}(j)}, X_{I_{2}(j)}\right) \approx \mathbb{E}\left[X_{I_{1}(j)}\right] \cdot \mathbb{E}\left[X_{I_{2}(j)}\right] .
$$

No error corresponds to a split of $X_{I \cup\{j\}}$ into $X_{I_{1}(j)}$ and $X_{I_{2}(j)}$ such that $\operatorname{Cov}\left(X_{I_{1}(j)}, X_{I_{2}(j)}\right)$ is zero. The factors should thus be as uncorrelated as possible.

ii. Choosing $F_{2}(x)=\sqrt{x}$ leads to the $n$ th-order Hilbert approximation.

In this case, we set

$$
\mathbb{E}\left[X_{I \cup\{j\}}\right]=\sqrt{\mathbb{E}\left[X_{I_{1}(j)}\right]} \cdot \sqrt{\mathbb{E}\left[X_{I_{2}(j)}\right]} \cdot \cos \phi \approx \sqrt{\mathbb{E}\left[X_{I_{1}(j)}\right]} \cdot \sqrt{\mathbb{E}\left[X_{I_{2}(j)}\right]},
$$

for $j \notin I$. The angle $\phi$ is defined via the scalar product $\langle R, S\rangle:=\mathbb{E}[R S]$ for $R, S \in \mathcal{L}^{2}$ in the usual Hilbert space. The split is good whenever the angle $\phi$ between $X_{I_{1}(j)}$ and $X_{I_{2}(j)}$ is close to $0^{\circ}$. This corresponds to highly dependent factors.

A naive single split partitions the set $I \cup\{j\}$ into the subsets $I_{1}(j)=I$ and $I_{2}(j)=\{j\}$. Note that the sets are enumerated and, thus, the naive single split is uniquely determined. This approach may not always be ideal, if small or strong dependence of the factors is desired in order to keep the error of the approximation small. Hence, we sketch an alternative approach that is based on a measurement of the nodes' distances in the graph.

The graph structure is described by the adjacency matrix $A \in \mathbb{R}^{N \times N}$, i.e., there exists a link between $i, j \in\{1,2, \ldots, N\}$, if $a_{i j}=a_{j i}=1$. If two agents are not directly connected, they might be connected indirectly. We define $\pi_{i j}$ as 
the length of the shortest path in the graph that connects two agents $i \neq j$, i.e.,

$$
\pi_{i j}:=\min \left\{l \in \mathbb{N}: a_{i k_{1}}=a_{k_{1} k_{2}}=a_{k_{2} k_{3}}=\cdots=a_{k_{l-1} j}=1\right\} .
$$

We set $\Pi=\left(\pi_{i j}\right)_{i, j=1,2, \ldots, N}$ with $\pi_{i i}=0$ for all $i=1,2, \ldots, N$. The splitting algorithm is described by the following pseudocode.

Algorithm 3.14.

INPUT An index set $I$ with $|I|=n$ and $j \notin I$ is given.

OUTPUT The output is a partition $I_{1}(j), I_{2}(j)$ of $I \cup\{j\}$ constructed as follows.

Step 1. Consider a set of partitions $J_{1}(j), J_{2}(j)$ of $I \cup\{j\}$.

Step 2. Choose some $\alpha \in(0,1)$ and calculate for each partition the following distance measure:

$$
m_{\alpha}\left(J_{1}(j), J_{2}(j)\right):=\sum_{i \in J_{1}(j)} \sum_{i^{\prime} \in J_{2}(j)} \alpha^{\pi_{i i^{\prime}}} .
$$

Step 3. We consider four alternative choices.

a. Minimal single split:

We choose the partition $I_{1}(j), I_{2}(j)$ with $\left|I_{2}(j)\right|=1$ that minimizes $m_{\alpha}$.

b. Maximal single split:

We choose the partition $I_{1}(j), I_{2}(j)$ with $\left|I_{2}(j)\right|=1$ that maximizes $m_{\alpha}$.

c. Minimal equal split:

We choose the partition $I_{1}(j), I_{2}(j)$ with $\left|I_{1}(j)\right|=\left|I_{2}(j)\right|$, if $n+1$ is even, or $\left|I_{1}(j)\right|=\left|I_{2}(j)\right|+1$, if $n+1$ is odd, that minimizes $m_{\alpha}$.

d. Maximal equal split:

We choose the partition $I_{1}(j), I_{2}(j)$ with $\left|I_{1}(j)\right|=\left|I_{2}(j)\right|$, if $n+1$ is even, or $\left|I_{1}(j)\right|=\left|I_{2}(j)\right|+1$, if $n+1$ is odd, that maximizes $m_{\alpha}$.

Observe that large values of our distance measure $m_{\alpha}$ correspond to small graph distances. In line with our heuristic arguments above, we thus apply choices a. and $\mathrm{c}$. to the independent approximation and choices b. and d. to the Hilbert approximation.

Example 3.15. To illustrate higher order mean-field approximations, we consider two different networks: the network with adjacency matrix A defined in Figure 1 with fixed infection rate $\beta=1 / 2$ and curing rate $\delta=2.01$, and the network with adjacency matrix $B$ defined in Figure 4 with $\beta=\delta=1 / 2$. For both networks, nodes 1, 3 and 7 are initially infected. Figure 5 displays the evolution of the aggregate infection probability of initially healthy nodes, i.e., $\sum_{j \in\{2,4,5,6\}} z_{j}^{(n)}$, for the original Markov chain together with mean-field approximations of order $n=2,3,4$ in both networks under the different splitting choices. We observe 


$$
B:=\left(\begin{array}{lllllll}
0 & 1 & 0 & 0 & 1 & 0 & 0 \\
1 & 0 & 1 & 1 & 0 & 0 & 0 \\
0 & 1 & 0 & 1 & 0 & 0 & 0 \\
0 & 1 & 1 & 0 & 0 & 0 & 0 \\
1 & 0 & 0 & 0 & 0 & 1 & 1 \\
0 & 0 & 0 & 0 & 1 & 0 & 1 \\
0 & 0 & 0 & 0 & 1 & 1 & 0
\end{array}\right)
$$

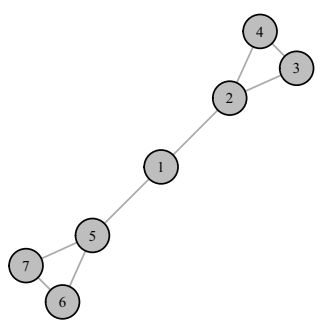

FIGURE 4: A network with $N=7$ nodes and spectral radius $\hat{\mu} \approx 2.3429$.

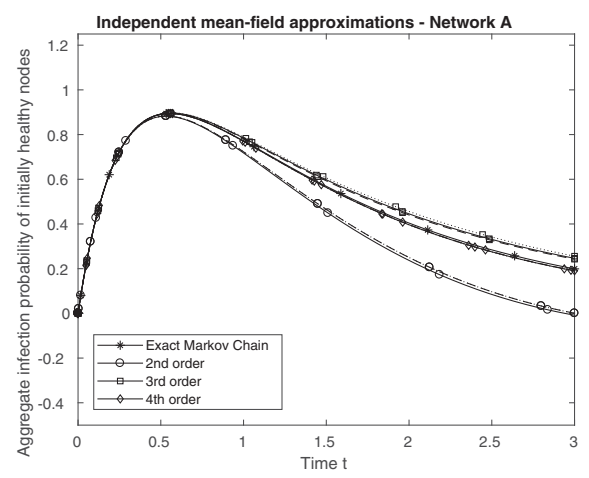

(A) Alg. 3.14 a. (dashed), c. (dotted)

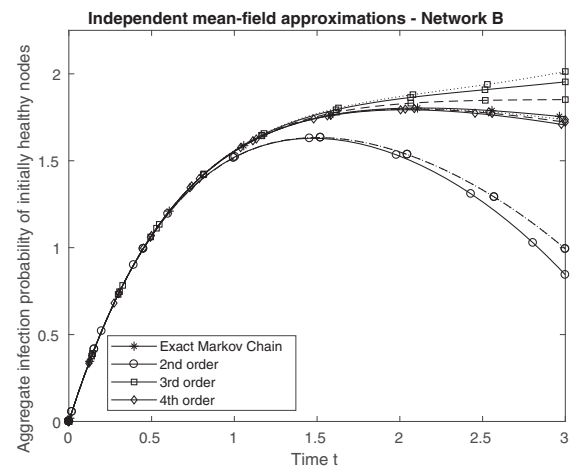

(c) Alg. 3.14 a. (dashed), c. (dotted)

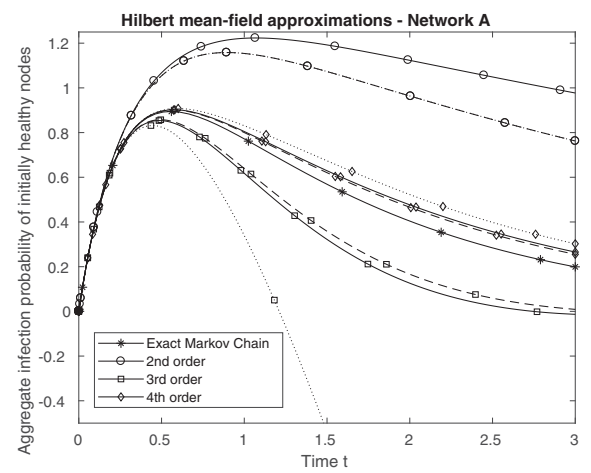

(B) Alg. 3.14 b. (dashed), d. (dotted)

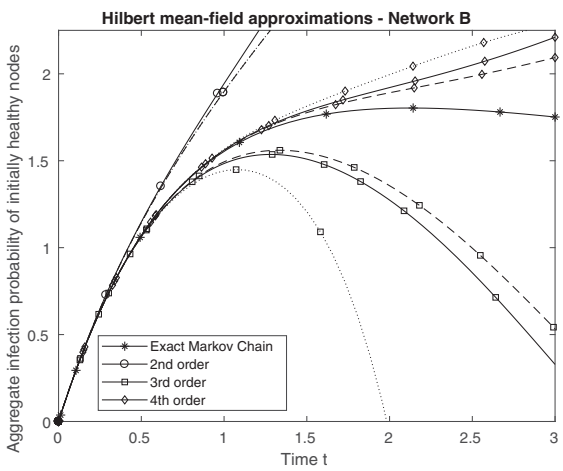

(D) Alg. 3.14 b. (dashed), d. (dotted)

FIGURE 5: Aggregate infection probability of initially healthy nodes. Solid lines represent naive single split approximations; dashed lines correspond to the results of Algorithm $3.14(\alpha=0.5)$ under a single split; dotted lines represent the results of Algorithm $3.14(\alpha=0.5)$ under an equal split.

that the higher the order of the mean-field approximation, the better is the approximation. In examples $(A),(B)$ and $(C)$, a fourth-order mean-field approximation already provides a reasonably good fit to the exact infection probabilities. 


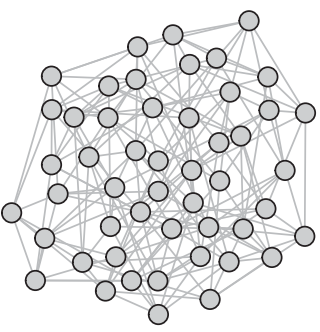

(A) Homogeneous

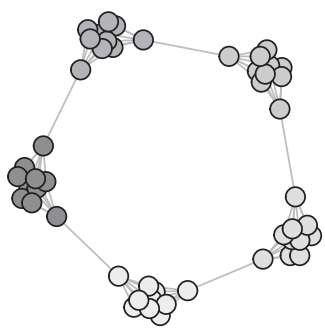

(B) Clustered

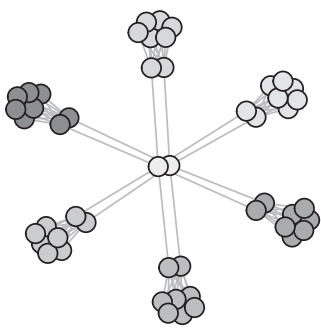

(C) Star-shaped

FIGURE 6: Stylized regular network scenarios. Quantities that are kept constant are the number of nodes $N=50$ and the degree of each node $D=7$. In particular, this yields a constant total number of edges $N D / 2=175$ and a constant spectral radius $\hat{\mu}=D=7$.

However, increasing the order of the approximation, also substantially increases its computational cost. In comparison to the naive single split, the optimized single split of Algorithm 3.14 leads to moderate improvements in all four cases. A split into subsets of equal size, however, impairs the approximation quality ${ }^{3}$ in the cases $(A),(B)$ and $(D)$. In contrast to that, in part $(C)$, the fourth-order equal split performs slightly better than the optimized single split. A possible reason for this is the special distance structure for subsets of size three in the network B. This indicates that the structure of the network topology is key to finding a splitting algorithm. Future research should investigate this issue further, analyze how Algorithm 3.14 might be improved, e.g., by choosing a better distance measure instead of $m_{\alpha}$, and how computational cost and precision can be optimally balanced.

\section{CASE STUdies}

\subsection{Model setting}

In this section, we compute the expected insurance losses for different insurance contracts in numerical case studies. We consider three network topologies, illustrated in Figure 6. All networks consist of $N=50$ agents or nodes which are all connected to $D=7$ other nodes. This means that the degree of each node is 7. The number of edges in each of the networks equals $N D / 2=175$, and the spectral radius of the three corresponding adjacency matrices is $\hat{\mu}=D=7$.

A. Homogeneous network: The first network that we consider consists of agents of the same type that are homogeneously connected to each other. The corresponding adjacency matrix of this and the other two considered networks can be found in Appendix D. In the homogeneous cyber networks, there is no hierarchy of nodes in terms of data flow and related cyber threats.

B. Clustered network: The key feature of the clustered network is that agents form groups that are closely connected. In contrast, agents within a cluster are less connected to agents from other clusters. This structure might be a 


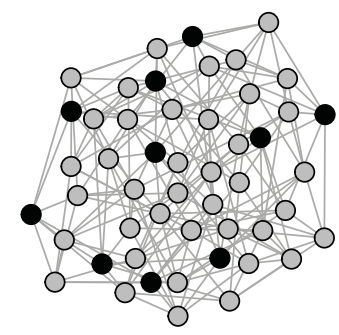

(A) Homogeneous

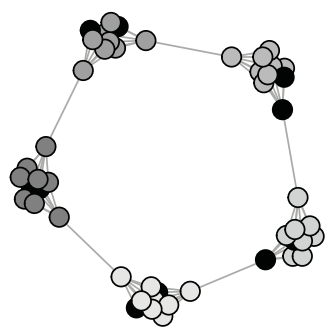

(B) Clustered

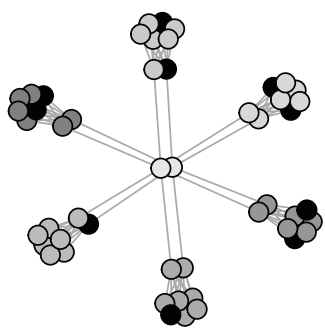

(c) Star

FIGURE 7: Infection scenario: The black nodes $(3,5,13,15,23,25,33,35,43,45)$ are initially infected.

more realistic model of cyber networks than a homogeneous network: Consider, e.g., different firms or divisions that are internally densely connected, but less densely connected with other firms or divisions. Qualitatively, the model captures different levels of security for internal and external connections.

C. Star-shaped network: Unlike the clustered network, the star-shaped cyber network possesses a clear hierarchy between central nodes that provide a hub for the data flow, and a number of periphery clusters that are internally homogeneously connected, but apart from the connections via the hub isolated from agents in other clusters.

The cyber networks are the channel for the spread of the cyber infection that makes agents vulnerable to cyber attacks. Cyber attacks affect all vulnerable agents at times that are modeled by a point process. The incurred losses are random. We will provide the parameters of the numerical case studies below.

In this setting, we compute the expected losses for three types of contracts: proportional insurance, XL insurance, and Cat-XL insurance. The functions that map physical losses to insured losses were described in equation (3) and Example 3.2. Exact expected contract losses can be computed according to (2). We will apply the mean-field approximation to estimate this quantity.

In our numerical case studies, we use the following parameters. The parameters of the infection dynamics (1) are $\beta=0.5$ and $\delta=\beta \cdot \hat{\mu}+0.01=3.51$. Initially, 10 nodes, i.e., $20 \%$ of all nodes, are infected. The location of the initially infected nodes is shown in Figure 7. These were sampled from an initial infection that is uniformly distributed across the network. Cyber attacks that cause losses at infected, i.e., vulnerable nodes occur at the jumps of a homogeneous Poisson process with rate $\lambda=3$. A cyber event causes a loss at each vulnerable node that is exponentially distributed with mean $\mu=2$, i.e., with parameter $1 / 2$. For the insurance contracts, we choose a policy period $T=3$.

For the numerical simulation, we exploit the mean-field approximation to analyze the spread process. In order to generate the losses due to cyber attacks, we use a simple Monte-Carlo approach. We simulate 100,000 sample paths of the homogeneous Poisson process and corresponding random losses 
in the time interval $[0, T]$. For each simulation we compute the integrand of equation (5). Averaging over these results yields our Monte-Carlo estimator of the expected aggregate losses of the reinsurance company; its standard error is stated in brackets.

\subsection{Model results}

We comment on the results of the simulation.

4.2.1. Infection probabilities and mean-field approximations. We first analyze the spread process of the cyber infection for the three different network structures. At this stage, we do not consider any cyber losses. We consider the independent approximation with naive single split. The aggregate infection probability of initially healthy nodes is a measure of the strength of the infection, i.e.,

$$
A P_{(n)}^{h}(t):=\sum_{i \in \mathcal{H}} z_{i}^{(n)}(t)
$$

where $\mathcal{H}$ denotes the set of initially healthy nodes and $n$ is the order of the meanfield approximation.

Figure 8 displays the approximation results for $n=1,2,3$, 4: First, for all networks, the fourth-order mean-field approximation and the third-order approximation are reasonably close to each other. This indicates that a mean-field approximation of order four provides a relatively good proxy for the exact probability of infection. Second, we find that the infection probability in the homogeneous network tends to be the highest (for $t>0.5$ ), while the star exhibits the lowest infection probability. This result will be of key importance for the expected losses of a reinsurance company.

4.2.2. Expected aggregate losses of the insurance company. We now study cyber losses.

Proportional reinsurance: For simplicity, we consider full insurance; other percentages lead to similar results. The results are shown in Table 1 . These indicate that the homogeneous network constitutes the highest risk, while the star network exhibits the lowest average losses.

$X \boldsymbol{L}$ : In this case, we assume that the limit per loss is equal to 2 . We consider only the lowest tier with priority 0 . Using the notation introduced in Example 3.2, the random insurance losses are $\sum_{i=1}^{N} \hat{L}_{i}(t) X_{i}(t)$ with $\hat{L}_{i}(t)=L_{i}(t)^{+}-\left(L_{i}(t)-2\right)^{+}=\min \left\{L_{i}(t), 2\right\}$. Apart from the modified losses $\hat{L}_{i}$ instead of $L_{i}$, we are again in the situation of a proportional contract. As expected, the numerical results in Table 2 show that the homogeneous network produces the highest expected losses, while the losses are lowest in the star network. 


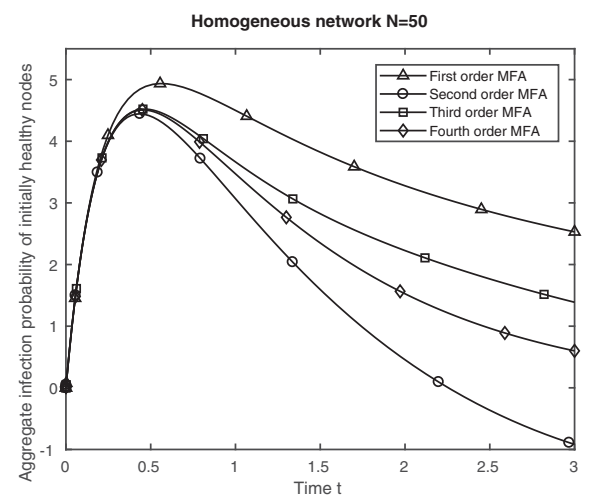

(A) $A P_{(n)}^{h}(t)$

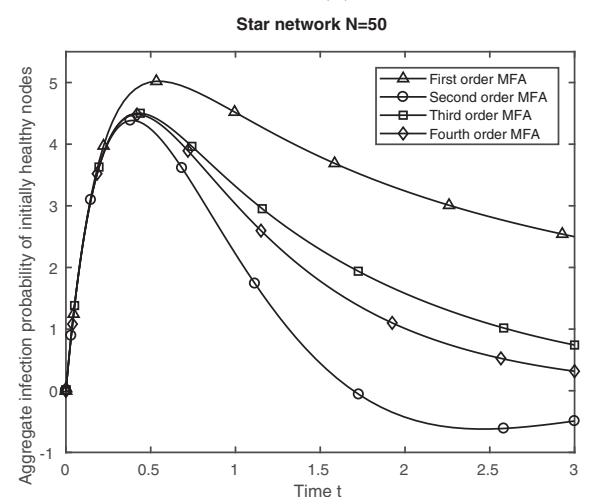

(C) $A P_{(n)}^{h}(t)$

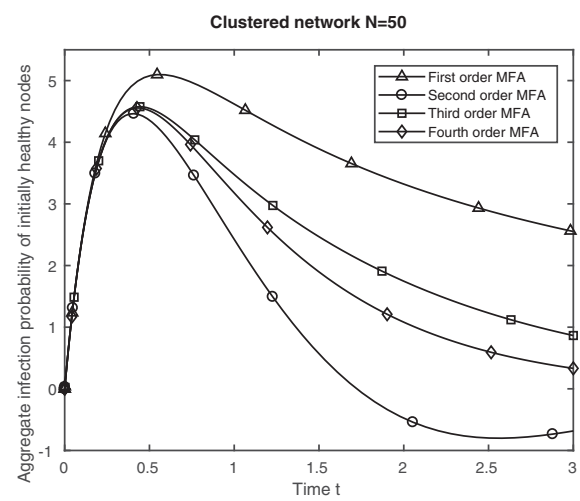

(B) $A P_{(n)}^{h}(t)$

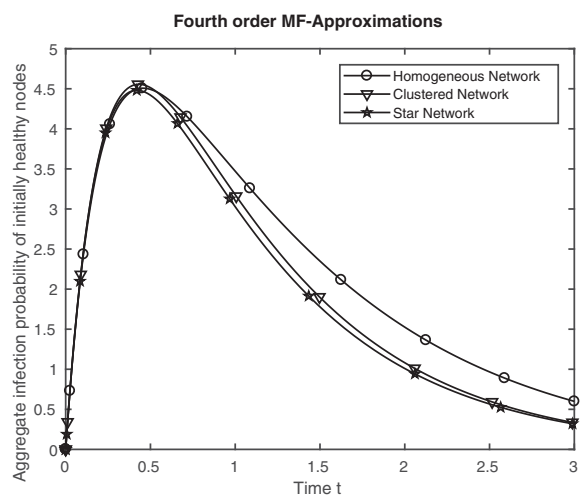

(D) $A P_{(4)}^{h}(t)$

FIGURE 8: Mean-field approximations of $\operatorname{order} n=1,2,3,4$ for the aggregate infection probability of initially healthy nodes in the homogeneous (A), clustered (B) and star network (C). Part (D) compares the fourth-order mean-field approximations for the different network structures.

TABLE 1

PROPORTIONAL INSURANCE.

\begin{tabular}{lccc}
\hline \hline Losses: Total Coverage & Homogeneous & Clustered & Star \\
\hline First-order MFA & $96.4671(0.1039)$ & $97.6170(0.1105)$ & $96.5425(0.1095)$ \\
Second-order MFA & $51.4911(0.0836)$ & $39.7776(0.0797)$ & $39.4127(0.0782)$ \\
Third-order MFA & $77.8349(0.0943)$ & $70.6588(0.0901)$ & $68.0767(0.0883)$ \\
Fourth-order MFA & $68.0676(0.0890)$ & $61.3693(0.0855)$ & $59.9005(0.0843)$ \\
\hline \hline
\end{tabular}

Cat-XL: Finally, we consider an excess of loss per event contract. We consider coverage of the lowest tier, i.e., a priority 0 , and choose a cover of 60 . Of course, the numerical results in Table 3 exhibit again the same ordering of the networks in terms of risk. But, more importantly, the numerical analysis 
TABLE 2

EXCESS OF LOSS PER RISK (XL).

\begin{tabular}{lccc}
\hline \hline Losses: $X L$ & Homogeneous & Clustered & Star \\
\hline First-order MFA & $60.9795(0.0684)$ & $61.7036(0.0692)$ & $61.0247(0.0686)$ \\
Second-order MFA & $32.5475(0.0522)$ & $25.1401(0.0497)$ & $24.9105(0.0488)$ \\
Third-order MFA & $49.2010(0.0589)$ & $44.6618(0.0563)$ & $43.0300(0.0552)$ \\
Fourth-order MFA & $43.0265(0.0556)$ & $38.7894(0.0534)$ & $37.8615(0.0526)$ \\
\hline \hline
\end{tabular}

TABLE 3

EXCESS OF LOSS PER EVENT (CAT-XL).

\begin{tabular}{lccc}
\hline \hline Losses: Cat-XL & Homogeneous & Clustered & Star \\
\hline$d=1$ & $169.6693(0.5733)$ & $166.6429(0.5638)$ & $165.9828(0.5616)$ \\
$d=2$ & $64.5432(0.3047)$ & $56.5714(0.2942)$ & $54.9458(0.2906)$ \\
$d=3$ & $52.4555(0.2867)$ & $44.0598(0.2796)$ & $42.3615(0.2764)$ \\
$d=4$ & $59.2664(0.8249)$ & $52.8354(0.7905)$ & $51.0151(0.7765)$ \\
\hline \hline
\end{tabular}

shows very clearly that the first-order mean-field approximation is not able to capture this type of contract. Due to its non-linearity, this is not surprising, but the extreme size of the error could not be expected a priori. This demonstrates that higher order mean-field approximations are necessary for the computation of the expected insurance losses and are thus also needed for pricing contracts within our model setting.

To be more specific, the contract function of the Cat-XL is given by

$$
f^{\mathrm{Cat}-\mathrm{XL}}(L(t) \circ X(t))=g^{\mathrm{Cat}-\mathrm{XL}}\left(\Lambda^{\Sigma}(L(t) \circ X(t))\right),
$$

where $\Lambda^{\Sigma}\left(x_{1}, \ldots, x_{N}\right):=\sum_{i=1}^{N} x_{i}$, and $g^{\text {Cat-XL }}(y):=(y)^{+}-(y-60)^{+}=$ $\min (y, 60)$. We follow Algorithm 3.4 and approximate the non-linear claim function $g^{\text {Cat-XL }}$ on a compact interval by a polynomial of chosen degree $d$.

- Compact approximation interval. We choose $\epsilon=0.05$ and determine a constant $u \in \mathbb{R}_{+}$such that

$$
P\left(\Lambda^{\Sigma}(L)>u\right) \leq \epsilon .
$$

We use the compact interval $[0, u]$ for the polynomial approximation. Setting $S_{t}:=\Lambda^{\Sigma}(L(t))=\sum_{i=1}^{N} L_{i}(t)$, we define $u_{t}:=F_{S_{t}}^{-1}(1-\epsilon)$. Since $S_{t}$ is independent of $t$ and Gamma-distributed with parameters $N=50$ and $\mu=2$, we obtain $u=u_{t} \approx 124.3412$.

- Polynomial approximation. For degrees $d \in\{1,2,3,4\}$, we determine the best uniform approximation of $g^{\mathrm{Cat}-\mathrm{XL}}$ on $[0, u]$. Figure 9 depicts the resulting polynomial approximations for $d \in\{1,2,3,4\}$. The error $e_{d}\left(g^{\mathrm{Cat}-\mathrm{XL}}\right)=$ $\left\|g^{\mathrm{Cat}-\mathrm{XL}}-p_{d}\right\|_{\infty,[0, u]}$ of the approximations on the interval is $e_{1}\left(g^{\mathrm{Cat}-\mathrm{XL}}\right)=$ 


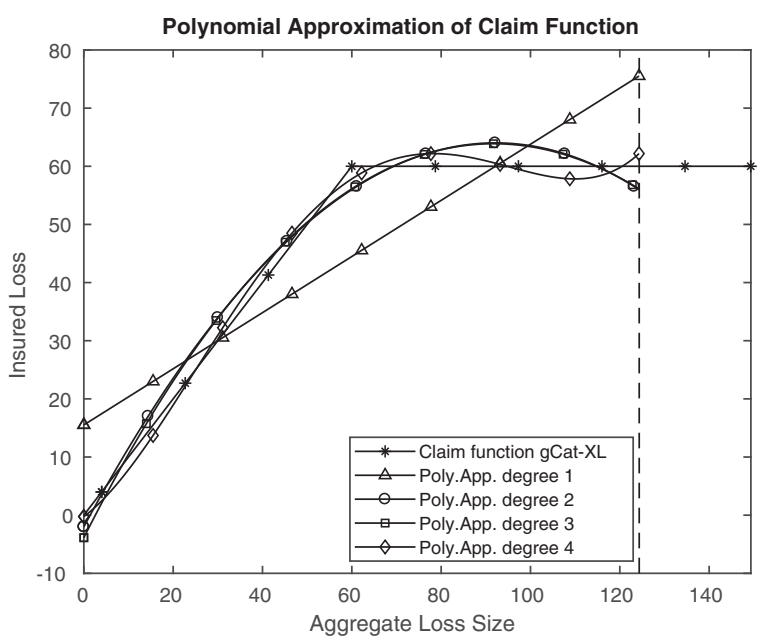

FIGURE 9: Best uniform polynomial approximations of $g^{\text {Cat-XL }}$ for different polynomial degrees in the compact interval [0, 124.3412].

$15.5189, e_{2}\left(g^{\mathrm{Cat}-\mathrm{XL}}\right)=4.0187, e_{3}\left(g^{\mathrm{Cat}-\mathrm{XL}}\right)=3.8741$ and $e_{4}\left(g^{\mathrm{Cat}-\mathrm{XL}}\right)=$ 2.1740 .

According to Section 3.1, the total $L_{1}$-error is bounded from above by $E_{d}:=$ $e_{d}\left(g^{\mathrm{Cat}-\mathrm{XL}}\right)+60 \cdot \epsilon$, which yields $E_{1} \approx 18.5189, E_{2} \approx 7.0187, E_{3} \approx 6.8741$ and $E_{4} \approx 5.1740$. We combine the polynomial approximations of $g^{\text {Cat-XL }}$ with the fourth-order independent mean-field approximation with naive single split. The fourth-order approximation is more accurate for lower order moments, but the linear polynomial approximation is of such a low quality that the resulting error is very large. In particular, a first-order mean-field approximation is not able to deliver appropriate results for the Cat-XL. The expected losses are given in Table 3. We reduced the number of Monte-Carlo samples to 10,000 for $d=$ $1,2,3$ and to 1,000 for $d=4$ due to the higher computational complexity.

The fact that the first-order approximation performs very badly can be seen by comparing the result to full insurance in Table 1. Clearly, full insurance should provide an upper bound on the Cat-XL. The polynomial approximation of degree 1 of the Cat-XL is, however, far larger.

Let us finally mention that we considered insurance of all agents in the network and an initial distribution of the infection that was uniformly sampled across the network. This implied a clear ranking of the three network structures in terms of the risk. The spread of the infection will be different, if specific nodes - e.g., the core nodes of the star network - are the origin of the infection. Our model is capable of identifying critical nodes from which the infection spreads. The effect can explicitly be quantified. Another interesting analysis concerns the elimination of links and its impact on aggregate losses. Thereby, connections can be identified (and potentially modified) that are critical for the spread of the infection. 
Remark 4.1. The implementation of the suggested methodology is based on several approximations that lead to approximation errors. First, the claim function is approximated by polynomials. Second, not the exact ODE system is solved, but only a mean-field approximation. Third, further sources of approximation errors are the numerical ODE solver and the Monte-Carlo simulation of the processes $M$ and $L$. This needs to be taken into account when cyber insurance contracts are evaluated.

\section{CONCLUSION}

We developed a model of cyber losses that are triggered by two underlying risk processes. First, a cyber infection spreads in a network, modeled by an interacting Markov process. Second, infected, i.e., vulnerable agents incur losses due to cyber attacks that occur according to a point process. Due to the large dimension of the system, the computation of expected aggregate insurance losses and pricing of cyber contracts is extremely challenging. We constructed a polynomial approximation for claim functions and higher order mean-field approximations that make these problems tractable. We demonstrated that for nonlinear claim functions, higher order polynomial approximations and mean-field approximations are indispensable. We also showed that, if the initial infection is uniformly distributed and all agents in the network are insured, homogeneous networks are the most risky and star networks the least. Our techniques can also be applied to identify critical initial infections and critical links in networks that augment expected losses. A key role is played by the network topology. While this paper focuses on fixed undirected graphs, future research could investigate more realistic structures such as directed or random graphs.

\section{NOTES}

1. On the computing cluster that we used, we were able to numerically solve the coupled system of ODEs for networks with $N \leq 13$ agents.

2. Similar results of decreasing error are found for decreasing infection rate $\beta$ and spectral radius $\hat{\mu}$.

3. Obviously, the optimized single and equal split coincide for the second-order approximations, since sets of cardinality three are split in this case.

\section{REFERENCES}

Bezuidenhout, C. and Grimmett, G. (1990) The critical contact process dies out. The Annals of Probability, 18(4), 1462-1482.

Bielecki, T.R. and RUtKowski, M. (2004) Credit Risk: Modeling, Valuation and Hedging. Springer Finance. Berlin, Heidelberg: Springer-Verlag.

BoguÑÁ, M. and PASTOR-SATORRAS, R. (2002) Epidemic spreading in correlated complex networks. Physical Review E, 66, 047104.

BöHME, R. (2005) Cyber-insurance revisited. 4th Annual Workshop on the Economics of Information Security, WEIS 2005, Harvard University, Cambridge, MA, USA, June 1-3, 2005. 
Bolot, J. and Lelarge, M. (2008) A new perspective on internet security using insurance. In IEEE INFOCOM 2008 - The 27th Conference on Computer Communications, pp. 71-75. Piscataway, NY: The Institute of Electrical and Electronics Engineers.

BRÉmaud, P. (1981) Point Processes and Queues: Martingale Dynamics. Springer Series in Statistics. New York, Heidelberg, Berlin: Springer-Verlag.

BURNECKI, K., JANCZURA, J. and WERON, R. (2011) Building loss models. In Statistical Tools for Finance and Insurance (eds. P. Cizek, W.K. Härdle and R. Weron), pp. 293-328. Berlin, Heidelberg: Springer-Verlag.

CAtor, E. and VAN Mieghem, P. (2012) Second-order mean-field susceptible-infected-susceptible epidemic threshold. Physical Review E, 85(5), 056111.

CAtOR, E. and VAN Mieghem, P. (2014) Nodal infection in Markovian susceptible-infectedsusceptible and susceptible-infected-removed epidemics on networks are non-negatively correlated. Physical Review E, 89(5), 052802.

DURRETT, R. and LiU, X.-F. (1988) The contact process on a finite set. The Annals of Probability, 16(3), 1158-1173.

GANDEL, S. (2015) Lloyd's CEO: Cyber attacks cost companies $\$ 400$ billion every year. Fortune. 23 January 2015. Available at: http://fortune.com/2015/01/23/cyber-attack-insurance-lloyds/.

GieseCKE, K. and Weber, S. (2004) Cyclical correlations, credit contagion, and portfolio losses. Journal of Banking and Finance, 28(12), 3009-3036.

GiESECKE, K. and WEBER, S. (2006) Credit contagion and aggregate losses. Journal of Economic Dynamics and Control, 30(5), 741-767.

HILlE, E. (1968) Lectures on Ordinary Differential Equations. Addison-Wesley Series in Mathematics. Reading, MA: Addison-Wesley Pub. Co.

Jacobsen, M. (2006) Point Process Theory and Applications: Marked Point and Piecewise Deterministic Processes. Probability and Its Applications. Boston: Birkhäuser.

JACOD, J. (1975) Multivariate point processes: Predictable projection, radon-nikodym derivatives, representation of martingales. Zeitschrift für Wahrscheinlichkeitstheorie und Verwandte Gebiete, 31(3), 235-253.

LAST, G. and BRANDT, A. (1995) Marked Point Processes on the Real Line: The Dynamic Approach. Probability and its Applications. New York: Springer-Verlag.

LiggetT, T.M. (1985) Interacting Particle Systems. Grundlehren der Mathematischen Wissenschaften, Vol. 276. New York: Springer-Verlag.

LigGeTt, T.M. (1999) Stochastic Interacting Systems: Contact, Voter and Exclusion Processes. Grundlehren der Mathematischen Wissenschaften, Vol. 324. Berlin: Springer-Verlag.

Marotta, A., Martinelli, F., NAnNi, S. and Yautsiukhin, A. (2015) A survey on cyberinsurance. Technical Rep. IIT TR-17/2015. Istituto di Informatica e Telematica, Consiglio Nazionale delle Richerce, Pisa.

MatA, A.S. and FerReirA, S.C. (2013) Pair quenched mean-field theory for the susceptibleinfected-susceptible model on complex networks. Europhysics Letters, 103(4), 48003.

MATKOWSKi, J. (2012) Mean-value theorem for vector-valued functions. Mathematica Bohemica, 137(4), 415-423.

MountFord, T., Mourrat, J.-C., Valesin, D. and Yao, Q. (2016) Exponential extinction time of the contact process on finite graphs. Stochastic Processes and their Applications, 126(7), 1974-2013.

NowZARI, C., Preciado, V.M. and PAPPAS, G.J. (2016) Analysis and control of epidemics: A survey of spreading processes on complex networks. IEEE Control Systems Magazine, 36(1), 26 46.

PASTOR-SATORRAs, R. and VeSPignANi, A. (2001) Epidemic dynamics and endemic states in complex networks. Physical Review E, 63, 066117.

Pastor-Satorras, R., Castellano, C., Van Mieghem, P. and Vespignani, A. (2015) Epidemic processes in complex networks. Reviews of modern physics, 87(3), 925.

ProtTER, P.E. (2004) Stochastic Integration and Differential Equations. Applications of Mathematics, Vol. 21. Berlin, Heidelberg: Springer-Verlag.

SCHWARTZ, G.A. and SASTRY, S.S. (2014) Cyber-insurance framework for large scale interdependent networks. In Proceedings of the 3rd International Conference on High Confidence Networked Systems, pp. 145-154. New York: The Association for Computing Machinery. 
Swiss Re Institute (2017) Cyber: Getting to grips with a complex risk. Sigma, 2017(1), 1-40.

Swiss Re/IBM (2016) Cyber: In search of resilience in an interconnected world. Expertise Publication. Available at: http://www.swissre.com/library/archive/Demand_for_cyber_insurance_on the_rise_joint_Swiss_Re_IBM_study_shows.html.

TAYlor, M.E. (2011) Partial Differential Equations I: Basic Theory. Applied Mathematical Sciences, Vol. 115, 2nd ed. New York: Springer-Verlag.

Teschl, G. (2012) Ordinary Differential Equations and Dynamical Systems. Graduate Studies in Mathematics, Vol. 140. Providence: American Mathematical Society.

Van Mieghem, P. (2011) The N-intertwined SIS epidemic network model. Computing, 93(24), 147-169.

VAn Mieghem, P., OMIC, J. and KoolJ, R. (2009) Virus spread in networks. IEEE/ACM Transactions on Networking, 17(1), 1-14.

Van Mieghem, P. and VAN DE Bovenkamp, R. (2015) Accuracy criterion for the mean-field approximation in susceptible-infected-susceptible epidemics on networks. Physical Review E, 91(3), 032812.

MATTHIAS A. FAHRENWALDT

Department of Actuarial Mathematics \& Statistics,

Maxwell Institute for Mathematical Sciences,

Heriot-Watt University,

Edinburgh EH14 4AS, UK

E-Mail:m.fahrenwaldt@hw.ac.uk

STEFAN WEBER (Corresponding author)

Institut für Mathematische Stochastik,

Leibniz Universität Hannover,

Welfengarten 1, 30167 Hannover, Germany

E-Mail:sweber@stochastik.uni-hannover.de

\section{KERSTIN WESKE}

Institut für Mathematische Stochastik,

Leibniz Universität Hannover,

Welfengarten 1, 30167 Hannover, Germany

E-Mail:weske@stochastik.uni-hannover.de 


\section{APPENDIX A. PROOFS}

\section{A.1. Proof of Theorem 3.7}

We define the two functions

$$
\begin{aligned}
& G_{1}(x):=(\beta A-\delta \mathbb{I}) x-\beta \operatorname{diag}(x) A x, \text { and } \\
& G_{2}(x):=(\beta A-\delta \mathbb{I}) x-\beta \operatorname{diag}(\sqrt{x}) A \sqrt{x},
\end{aligned}
$$

for $x \in \mathbb{R}^{N}$.

i. Existence. For $G_{1}$, the Picard-Lindelöf theorem (Theorem B.1) used in the proof of ii. also yields existence of a solution to (11).

For $G_{2}$, i.e., for the ODE describing the first-order Hilbert approximation, we use Theorem B.2 to prove existence of a solution. Define a non-linear map $\bar{G}_{2}: \mathbb{R}^{N} \rightarrow \mathbb{R}^{N}$ by $\bar{G}_{2}=(\beta A-\delta \mathbb{I}) x-\beta \operatorname{diag} \sqrt{|x|} A \sqrt{|x|}$, where the absolute value and square root are taken componentwise. Now consider the ODE system $\dot{X}(t)=\bar{G}_{2}(X(t))$. This enlarges the domain of definition of $\bar{G}_{2}$ compared to the original function in (13), $G_{2}$, but does not do any harm for initial conditions in $\mathbb{R}_{>0}^{N}$.

We now estimate the norm of $\bar{G}_{2}$. The matrix $A=\left(a_{i j}\right)_{1 \leq i, j \leq N}$ has zeros on the main diagonal so we find

$$
\operatorname{diag} \sqrt{|x|} A \sqrt{|x|}=\left(\sum_{j \neq 1} a_{1 j} \sqrt{\left|x_{1} x_{j}\right|}, \ldots, \sum_{j \neq N} a_{N j} \sqrt{\left|x_{N} x_{j}\right|}\right)^{\top} .
$$

With $\left|x_{i}\right| \leq\|x\|_{\infty}$ and noting that $a_{i j} \in\{0,1\}$, we have

$$
\sum_{j \neq i} a_{i j} \sqrt{\left|x_{i} x_{j}\right|} \leq \sum_{j \neq i}\|x\|_{\infty}=(N-1)\|x\|_{\infty} .
$$

Moreover, $\|A x\|_{\infty} \leq\|A\| \cdot\|x\|_{\infty}$ for the operator norm of $A$ with respect to the $l^{\infty}$-norm. Thus, overall,

$$
\left\|\bar{G}_{2}(x)\right\|_{\infty} \leq(\beta\|A\|+\delta+N-1)\|x\|_{\infty} .
$$

Now let $x_{0} \in \mathbb{R}^{N}$ and choose $r>0$. The previous inequality translates to

$$
\left\|\bar{G}_{2}(x)\right\|_{\infty} \leq C\left(\left\|x_{0}\right\|_{\infty}+r\right) .
$$

on $B_{r}\left(x_{0}\right)=\left\{x \in \mathbb{R}^{N}:\left\|x-x_{0}\right\|<r\right\}$ with $C=\beta\|A\|+\delta+N-1$. Then, consider the reciprocal fraction $M / r$ with $M=\sup _{x \in B_{r}\left(x_{0}\right)}\left\|\bar{G}_{2}(x)\right\|$

$$
\frac{M}{r} \leq \frac{C\left(\left\|x_{0}\right\|_{\infty}+r\right)}{r} .
$$

Thus,

$$
\frac{r}{M} \geq \frac{1}{C} \frac{r}{\left\|x_{0}\right\|_{\infty}+r}
$$

Theorem B.2 now shows that existence of a solution to (13) holds for any $T$ satisfying the inequality $T<r / M$. 
The global existence of a solution now follows from an iteration argument where in the $j$ th step we have initial condition $x_{0}^{(j)}$ and radius $r^{(j)}$. Start with any given initial condition $x_{0}^{(1)}=W(0)$ and set $r^{(1)}=\left\|x_{0}^{(1)}\right\|_{\infty}$. Then, by Theorem B.2 there is a solution to (13), $W$, on the time interval $\left(0, \frac{1}{2 C}\right]$. Repeat this process with the new initial condition $x_{0}^{(2)}=W\left(\frac{1}{2 C}\right)$ and with $r^{(2)}=\left\|x_{0}^{(2)}\right\|_{\infty}$. This will again have a solution on an interval of length at least $\frac{1}{2 C}$. Iterating this process and concatenating the solutions yields a solution on $[0, T]$ for arbitrary $T$.

ii. Uniqueness. Let $B_{R}(\mathbf{0})$ be the open ball of radius $R$ in $\mathbb{R}^{N}$ endowed with the Euclidean norm. We prove uniqueness using the Picard-Lindelöf theorem (Theorem B.1). Thus, we need to show that there exists a Lipschitz constant $L>0$ such that

$$
\left\|G_{1}\left(x_{1}\right)-G_{1}\left(x_{2}\right)\right\|_{2} \leq L\left\|x_{1}-x_{2}\right\|_{2}
$$

for all $x_{1}, x_{2} \in B_{R}(\mathbf{0})$. Then, Theorem B.1 states that the ODE has a unique solution on some small time interval $\left[0, t_{0}\right]$. Since the Lipschitz constant $L$ does not depend on time, the solution exists on the time interval $[0, \infty)$. The solution is smooth by Proposition 6.2 of Taylor (2011) so in particular belongs to $C\left([0, \infty), \mathbb{R}^{N}\right)$.

To prove the Lipschitz condition (A.1), first recall that the operator norm $\|A\|_{o p}$ of a matrix $A$ acting $\mathbb{R}^{N} \rightarrow \mathbb{R}^{N}$ endowed with the Euclidean norm is given by the spectral radius $\hat{\mu}$ of $A$. Thus, it follows that the operator norm of $\operatorname{diag}(x)$ is bounded by $\|x\|_{2}$. The claim now follows from a direct calculation. Let $x_{1}, x_{2} \in B_{R}(\mathbf{0})$. Then,

$$
\begin{aligned}
& \left\|G_{1}\left(x_{1}\right)-G_{1}\left(x_{2}\right)\right\|_{2} \\
= & \left\|(\beta A-\delta \mathbb{I}) x_{1}-\beta \operatorname{diag}\left(x_{1}\right) A x_{1}-(\beta A-\delta \mathbb{I}) x_{2}+\beta \operatorname{diag}\left(x_{2}\right) A x_{2}\right\|_{2} \\
= & \left\|(\delta \mathbb{I}-\beta A)\left(x_{1}-x_{2}\right)+\beta\left(\operatorname{diag}\left(x_{1}-x_{2}\right) A x_{1}+\beta \operatorname{diag}\left(x_{2}\right) A\left(x_{1}-x_{2}\right)\right)\right\|_{2} \\
\leq & \delta\left\|x_{1}-x_{2}\right\|_{2}+\beta\|A\|\left\|_{o p}\right\| x_{1}-x_{2}\left\|_{2}+\beta\right\| \operatorname{diag}\left(x_{1}-x_{2}\right)\|\|_{o p}\|A\|\left\|_{o p}\right\| x_{1} \|_{2} \\
& +\beta\left\|\operatorname{diag}\left(x_{2}\right)\right\|_{o p}\|A\|\left\|_{o p}\right\| x_{1}-x_{2} \|_{2} \\
= & \left(\delta+\beta \hat{\mu}(1+2 R)\left\|x_{1}-x_{2}\right\|_{2},\right.
\end{aligned}
$$

where we used the facts that $\left\|\operatorname{diag}\left(x_{1}-x_{2}\right)\right\|_{o p} \leq\left\|x_{1}-x_{2}\right\|_{2}$ and $\left\|x_{i}\right\|_{2}<R$ for $i=1,2$. iii. Sandwich property.

a. First, we prove $W(t) \geq \mathbf{0}$ for all $t \geq 0$. By definition, all components of $W(0)$ are non-negative. Now, by inspection of (13), we can immediately deduce that whenever a component, say $w_{i}$, at some time $t^{\prime}$ is zero, then its derivative $\dot{w}_{i}\left(t^{\prime}\right)$ is non-negative. Thus, the trajectory of $w_{i}(t)$ will be non-negative for some time by the fundamental theorem of calculus. By the continuity of the solution, it follows that $W(t) \geq \mathbf{0}$ for all $t \geq 0$.

b. Second, we prove the lower inequality for the exact dynamics, i.e., $W(t) \leq \mathbb{E}[X(t)]$ for all $t \geq 0$. The proof of the upper inequality $\mathbb{E}[X(t)] \leq V(t)$ is analogous. To begin with, we know that $W(0)=\mathbb{E}[X(0)]=V(0) \in[0,1]^{N}$ by definition and that $d W(t) / d t \leq d \mathbb{E}[X(t)] / d t$ (see equation (12)). Let $H(t):=\mathbb{E}[X(t)]-W(t)$, i.e., $h_{i}(t)=\mathbb{E}\left[X_{i}(t)\right]-w_{i}(t)$ for all $i=1, \ldots, N$. Then, $d H(t) / d t \geq \mathbf{0}$ for $t \geq 0$ and $H(0)=\mathbf{0}$. Now consider the interval $[0, t] \subset \mathbb{R}$ for $t>0$. From the mean-value theorem for vector-valued functions (cf. Matkowski, 2012), it follows that there exist 
constants $m_{1}, \ldots, m_{N} \in[0, t]$ such that:

$$
H(t)-H(0)=(t-0) \cdot\left(\frac{d h_{1}}{d t}\left(m_{1}\right), \ldots, \frac{d h_{N}}{d t}\left(m_{N}\right)\right)^{\top} \geq \mathbf{0} .
$$

Thus, we have that $H(t) \geq \mathbf{0}$ for all $t \geq 0$, which proves $W(t) \leq \mathbb{E}[X(t)]$ for all $t \geq 0$.

c. Third, we show that $V(t) \leq \mathbf{1}$ for all $t \geq 0$. By definition, all components of $V(0)$ are not larger than 1 . Now, by inspection of (11), we can immediately deduce that whenever a component at some time $t^{\prime}$ is 1 , e.g., $v_{i}\left(t^{\prime}\right)=1$, the reaction term vanishes so that $\dot{v}_{i}\left(t^{\prime}\right)=-\delta<0$. Thus, the function is pushed away from 1 toward 0 . By the continuity of the solution, it follows that $V(t) \leq \mathbf{1}$ for all $t \geq 0$.

iv. Stability. We apply Theorem B.3 to the function $G_{1}$. First, $V^{*} \equiv \mathbf{0}$ is an obvious fix-point of $G_{1}$. Second, the Jacobi matrix of $G_{1}$ at any point $V \in \mathbb{R}^{N}$ is given by

$$
J_{G_{1}}(V)=\beta A-\delta \mathbb{I}-\beta(\operatorname{diag}(V) A+\operatorname{diag}(A V)) .
$$

In particular, at the fix-point $V^{*} \equiv \mathbf{0}$, the Jacobi matrix reduces to

$$
J_{G_{1}}(\mathbf{0})=\beta A-\delta \mathbb{I}=-\delta(\mathbb{I}-\tau A),
$$

with $\tau=\beta / \delta . J_{G_{1}}(\mathbf{0})$ is real and symmetric and, thus, possesses only real eigenvalues. Let $\lambda_{1}, \ldots, \lambda_{N}$ denote the eigenvalues of $A$. Then, the eigenvalues of $J_{G_{1}}(0)$ are given by $-\delta\left(1-\tau \lambda_{i}\right)$ for $i=1, \ldots, N$. Hence, all eigenvalues of $J_{G_{1}}(0)$ are negative if and only if $\tau<1 / \max _{1 \leq i \leq N} \lambda_{i}=1 / \hat{\mu}=: \tau_{c}^{(1)}$. Thus, in this case, it follows from Theorem B.3 that the fix-point $V^{*} \equiv \mathbf{0}$ is exponentially stable. Finally, since $W(t) \leq V(t)$ (part iii.), it follows that the zero solution is also exponentially stable for (13).

\section{A.2. Proof of Theorem 3.9}

The difference $y_{i}(t)=\mathbb{E}\left[X_{i}(t)\right]-v_{i}(t)$ satisfies the ODE

$$
\begin{aligned}
\frac{d}{d t} y_{i}(t)= & -\delta y_{i}(t)+\beta\left(1-y_{i}(t)\right) \sum_{k=1}^{N} a_{k i} y_{k}(t) \\
& -\beta R_{i}(t)-\beta y_{i}(t) \sum_{k=1}^{N} a_{k i} v_{k}(t)-\beta v_{i}(t) \sum_{k=1}^{N} a_{k i} y_{k}(t),
\end{aligned}
$$

with initial condition $y_{i}(0)=0$. We want to apply Gronwall's inequality to the $l^{2}$-norm $\frac{1}{2}\|y(t)\|_{2}^{2}$. As a first step, we note that

$$
\begin{aligned}
\frac{d}{d t} \frac{1}{2} y_{i}(t)^{2}= & y_{i}(t) \cdot \frac{d}{d t} y_{i}(t) \\
= & -\delta y_{i}(t)^{2}+\beta y_{i}(t)\left(1-y_{i}(t)\right) \sum_{k=1}^{N} a_{k i} y_{k}(t) \\
& -\beta y_{i}(t) R_{i}(t)-\beta y_{i}(t)^{2} \sum_{k=1}^{N} a_{k i} v_{k}(t)-\beta y_{i}(t) v_{i}(t) \sum_{k=1}^{N} a_{k i} y_{k}(t) .
\end{aligned}
$$


Summing over all $i$, we obtain

$$
\begin{aligned}
\frac{d}{d t} \frac{1}{2}\|y(t)\|^{2}= & -\delta\|y(t)\|^{2}+\beta \sum_{i=1}^{N} y_{i}(t)\left(1-y_{i}(t)\right) \sum_{k=1}^{N} a_{k i} y_{k}(t) \\
& -\beta\langle y(t), R(t)\rangle-\beta \sum_{i=1}^{N} y_{i}(t)^{2} \sum_{k=1}^{N} a_{k i} v_{k}(t)-\beta \sum_{i=1}^{N} y_{i}(t) v_{i}(t) \sum_{k=1}^{N} a_{k i} y_{k}(t),
\end{aligned}
$$

where $\langle\cdot, \cdot\rangle$ denotes the standard inner product on $\mathbb{R}^{N}$.

We now estimate the terms on the right-hand side. Recall that $\mathbb{E}\left[X_{i}(t)\right]$ and $v_{i}(t)$ only take values in the interval $[0,1]$ so that also $\left|y_{i}(t)\right| \leq 1$. Denote by $|y(t)|$ the vector $\left(\left|y_{1}(t)\right|, \ldots,\left|y_{N}(t)\right|\right)$.

- We find that

$$
\begin{aligned}
\left|\beta \sum_{i=1}^{N} y_{i}(t)\left(1-y_{i}(t)\right) \sum_{k=1}^{N} a_{k i} y_{k}(t)\right| & \leq \beta \sum_{i=1}^{N}\left|y_{i}(t)\right| \sum_{k=1}^{N} a_{k i}\left|y_{k}(t)\right| \\
& \leq \beta\langle|y(t)|, A|y(t)|\rangle \\
& \leq \beta\|A\|\left\|_{o p}|| y(t)\right\|^{2} .
\end{aligned}
$$

- Clearly, $-\beta \sum_{i=1}^{N} y_{i}(t)^{2} \sum_{k=1}^{N} a_{k i} v_{k}(t) \leq 0$.

- It holds that $-\beta\langle y(t), R(t)\rangle \leq|\beta\langle y(t), R(t)\rangle| \leq \beta\|y(t)\|\|R(t)\| \leq \frac{\beta}{2}\|y(t)\|^{2}+\frac{\beta}{2}\|R(t)\|^{2}$

- As for the first term, we find

$$
\begin{aligned}
\left|\beta \sum_{i=1}^{N} y_{i}(t) v_{i}(t) \sum_{k=1}^{N} a_{k i} y_{k}(t)\right| & \leq \beta \sum_{i=1}^{N}\left|y_{i}(t)\right| \sum_{k=1}^{N} a_{k i}\left|y_{k}(t)\right| \\
& \leq \beta\langle|y(t)|, A|y(t)|\rangle \\
& \leq \beta\left\|A||_{o p}\right\| y(t) \|^{2} .
\end{aligned}
$$

As before, $\|A\|_{o p}$ denotes the operator norm of the matrix $A$; it is given by the largest eigenvalue $\hat{\mu}$ of $A$.

Collecting these estimates we arrive at

$$
\frac{d}{d t} \frac{1}{2}\|y(t)\|^{2} \leq\left(-\delta+2 \beta\|A\|_{o p}+\frac{\beta}{2}\right)\|y(t)\|^{2}+\frac{\beta}{2}\|R(t)\|^{2}
$$

or

$$
\frac{d}{d t}\|y(t)\|^{2} \leq\left(-2 \delta+4 \beta\|A\|_{o p}+\beta\right)\|y(t)\|^{2}+\beta\|R(t)\|^{2} .
$$

We now apply Gronwall's inequality in its differential form for which the sign of the term in brackets is immaterial. This yields

$$
\|y(t)\|^{2} \leq e^{\left(-2 \delta+4 \beta\|A\|_{o p}+\beta\right) t} \beta \int_{0}^{t}\|R(s)\|^{2} d s,
$$

as claimed. 


\section{APPENDIX B: BASIC ODE THEORY}

For convenience of the reader, this section contains the basic results from ODE theory used in the proofs of Appendix A. To begin with, we need the following two main results on existence and uniqueness (cf. (Taylor, 2011, Theorem 2.1) and (Hille, 1968, Theorem 2.4.1)).

Theorem B.1 (Picard-Lindelöf-Uniqueness). Consider the general ODE system

$$
\frac{d y}{d t}=F(t, y), \quad y\left(t_{0}\right)=y_{0}
$$

with $F: \operatorname{Dom}(F) \subset \mathbb{R}^{N+1} \rightarrow \mathbb{R}^{N}$ defined in a neighborhood of $\left(t_{0}, y_{0}\right)$. Let $y_{0} \in \mathcal{O}$ be an open subset of $\mathbb{R}^{n}$ and $I \subset \mathbb{R}$ an interval containing $t_{0}$. Suppose $F$ is continuous on $I \times \mathcal{O}$ and satisfies the Lipschitz condition

$$
\left\|F\left(t, y_{1}\right)-F\left(t, y_{2}\right)\right\| \leq L\left\|y_{1}-y_{2}\right\|,
$$

for $t \in I, y_{j} \in \mathcal{O}$. Then, equation (B.1) has a unique solution on some t-interval containing $t_{0}$.

Theorem B.2 (Peano-Existence). Suppose $F(y)$ is defined and continuous in $B_{r}\left(y_{0}\right)$ where $B_{r}\left(y_{0}\right)=\left\{y \in \mathbb{R}^{n}:\left\|y-y_{0}\right\|<r\right\}$ and suppose that $\|F(y)\| \leq M$ on $B_{r}\left(y_{0}\right)$. Then, the autonomous differential equation $\frac{d y}{d t}=F(y)$ with initial condition $y_{0}$ has at least one solution $y$ defined on the time interval $(-T, T)$ where $T<r / M$.

In addition to the key questions of existence and uniqueness, one is also interested in equilibria, i.e., fix-points $y^{*}$ with $F\left(t, y^{*}\right)=0$, and their stability, i.e., the behavior of solutions near them. For the autonomous differential system,

$$
\frac{d y}{d t}=F(y), \quad y(0)=y_{0},
$$

a fix-point $y^{*}$ is called exponentially stable, if there exist constants $\alpha, \epsilon, C>0$ such that for all $t \geq 0$

$$
\left|y(t)-y^{*}\right| \leq C e^{-\alpha t\left|y(0)-y^{*}\right|},
$$

for any $\left|y(0)-y^{*}\right| \leq \epsilon$ (Teschl, 2012, Chapter 6.5). Exponential stability is the strongest type of equilibrium stability and can be proven using the following basic result (Teschl, 2012, Theorem 6.10):

Theorem B.3. (Exponential stability via linearization). Suppose $F \in C^{1}$ has a fix-point $y^{*}$ and suppose that all eigenvalues of the Jacobian matrix at $y^{*}$ have negative real part. Then, $y^{*}$ is exponentially stable. 


\section{APPENDIX C: MATRIX FORM OF THE $n$ TH-ORDER MEAN-FIELD APPROXIMATION}

Let $M:=\sum_{k=1}^{n}\left(\begin{array}{l}N \\ k\end{array}\right)$ and denote the $n$ th-order mean-field approximation by

$$
z^{(n)}:=\left(z_{1}^{(n)}, z_{2}^{(n)}, \ldots, z_{N}^{(n)}, z_{12}^{(n)}, \ldots, z_{1 N}^{(n)}, \ldots, z_{1 \ldots n}^{(n)}, \ldots\right)^{\top} \in \mathbb{R}^{M},
$$

i.e., by the vector of solutions to the ODE system (16) in lexicographical order. Written in matrix form, (16) reads

$$
\dot{z}^{(n)}=\underbrace{\left(\beta A^{(n)}-\delta \operatorname{diag}(c)\right) z^{(n)}}_{\text {Linear term }}-\beta \underbrace{Q\left(z^{(n)} ; F, \text { Split }\right)}_{\text {Quadratic term }} .
$$

As in the first-order case, the matrix equation is given as the difference of a linear and a quadratic term. Note that the linear term is independent of the mean-field function $F$ and the chosen split, whereas the quadratic term crucially relies on these parameters. We now describe both terms in detail.

Linear term: The matrix $A^{(n)} \in \mathbb{R}^{M \times M}$ is a tridiagonal block matrix

$$
A^{(n)}:=\left[\begin{array}{cccccc}
D^{1,1} & U^{1,2} & & & & 0 \\
L^{2,1} & D^{2,2} & U^{2,3} & & & \\
& L^{3,2} & D^{3,3} & U^{3,4} & & \\
& & \ddots & \ddots & \ddots & \\
& & & L^{n-1, n-2} & D^{n-1, n-1} & U^{n-1, n} \\
0 & & & & L^{n, n-1} & D^{n, n}
\end{array}\right],
$$

with diagonal blocks

$$
\begin{aligned}
& D^{k, k} \in \mathbb{R}^{\left(\begin{array}{l}
N \\
k
\end{array}\right) \times\left(\begin{array}{l}
N \\
k
\end{array}\right)} \quad \text { with entries } \\
& D_{I, I}^{k, k}=A_{I, I}^{(n)}=-\sum_{i \in I} \sum_{j \in I} a_{i j}, \\
& D_{I, I \backslash\{j \cup\{j\}(i \in I, j \notin I)}^{k, k}=A_{I, I \backslash\{i\} \cup\{j\}(i \in I, j \notin I)}^{(n)}=a_{i j}, \\
& \text { for }|I|=k, \text { and } k=1, \ldots, n,
\end{aligned}
$$

upper diagonal blocks

$$
\begin{aligned}
& U^{k, k+1} \in \mathbb{R}^{\left(\begin{array}{c}
N \\
k
\end{array}\right) \times\left(\begin{array}{c}
N \\
k+1
\end{array}\right)} \quad \text { with entries } \\
& \qquad U_{I, I \cup\{j\}(j \notin I)}^{k, k+1}=A_{I, I \cup\{j\}(j \notin I)}^{(n)}=-\sum_{i \in I} a_{i j}, \\
& \text { for }|I|=k, k=1, \ldots, n-1,
\end{aligned}
$$


and lower diagonal blocks

$$
\begin{aligned}
& L^{k, k-1} \in \mathbb{R}^{\left(\begin{array}{c}
N \\
k
\end{array}\right) \times\left(\begin{array}{c}
N \\
k-1
\end{array}\right) \quad \text { with entries }} \\
& \qquad L_{I, I \backslash\{i\}(i \in I)}^{k, k-1}=A_{I, I \backslash\{i\}(i \in I)}^{(n)}=\sum_{j \in I} a_{i j}, \\
& \text { for }|I|=k, k=2, \ldots, n .
\end{aligned}
$$

The matrix $\operatorname{diag}(c) \in \mathbb{R}^{M \times M}$ is a diagonal matrix with vector $c \in \mathbb{R}^{M}$ on the diagonal. The vector indicates the cardinality of the underlying index set, i.e., $c_{I}=k$ if and only if $|I|=k$.

Quadratic term: The quadratic term $Q\left(z^{(n)} ; F\right.$, Split) depends on the mean-field function $F$ and on the chosen split $I \cup\{j\}=I_{1}(j) \cup I_{2}(j)$ (for all subsets $I \subseteq\{1, \ldots, N\}$ of size $|I|=n$ and all indices $j \in\{1, \ldots, N\}$ with $j \notin I)$. We denote the split in matrix form by $N$ pairs of matrices

$$
\left(\mathbb{I}_{1}(1), \mathbb{I}_{2}(1)\right), \ldots,\left(\mathbb{I}_{1}(N), \mathbb{I}_{2}(N)\right),
$$

where $\mathbb{I}_{\ell}(j) \in\{0,1\}^{M \times M}(\ell=1,2)$ is defined by its entries

$$
\left(\mathbb{I}_{\ell}(j)\right)_{I, J}:= \begin{cases}1, & \text { if }|I|=n, j \notin I, J=I_{\ell}(j), \\ 0, & \text { otherwise, }\end{cases}
$$

i.e., the row of the matrices $\mathbb{I}_{1}(j)$ and $\mathbb{I}_{2}(j)$ that corresponds to the set $I$ encodes the subset split of $I \cup\{j\}$, i.e., $I_{1}(j)$ and $I_{2}(j)$, respectively. Using this notation for the split, we can write the quadratic term in matrix form as

$$
Q\left(z^{(n)} ; F,\left(\mathbb{I}_{1}(j), \mathbb{I}_{2}(j)\right)_{j=1, \ldots, N}\right)=\sum_{j=1}^{N} \operatorname{diag}\left(\mathbb{I}_{1}(j) \cdot F\left(z^{(n)}\right)\right) \cdot C^{(n)}(j) \cdot \mathbb{I}_{2}(j) \cdot F\left(z^{(n)}\right),
$$

where

$$
F\left(z^{(n)}\right):=\left(F\left(z_{1}^{(n)}\right), F\left(z_{2}^{(n)}\right), \ldots, F\left(z_{N}^{(n)}\right), F\left(z_{12}^{(n)}\right), \ldots, F\left(z_{1 N}^{(n)}\right), \ldots, F\left(z_{1 \ldots n}^{(n)}\right), \ldots\right)^{\top} \in \mathbb{R}^{M},
$$

and for $j=1, \ldots, N$, the diagonal matrix $C^{(n)}(j) \in \mathbb{R}^{M \times M}$ is defined by its entries

$$
\left(C^{(n)}(j)\right)_{I, I}:= \begin{cases}\sum_{i \in I} a_{i j}, & \text { if }|I|=n, j \notin I \\ 0, & \text { otherwise. }\end{cases}
$$

Remark C.1 Plugging the derived expression for $Q\left(z^{(n)} ; F\right.$, Split) into equation (C.1), we immediately see that the matrix equations for the $n$ th-and first-order mean-field approximations ( $c f$. equations (11) and (13)) possess an analogous structure. Hence, the existence of a solution to the nth-order mean-field approximation (for the mean-field functions $F(x)=x$ and $F(x)=\sqrt{x}$ ) as well as its uniqueness (for $F(x)=x$ ) follow analogously to the proof of Theorem $3.7 i$. and ii., respectively.

Example C.2 As a concrete example, we consider the quadratic term for the naive single split. Recall that this split is defined by $I_{1}(j)=I$ and $I_{2}(j)=j$ for all possible subsets $I$ of size $n$ 
and indices $j \notin I$. In this case, the matrices $\mathbb{I}_{1}^{\text {single }}(j)$ become diagonal matrices, i.e.,

$$
\left(\mathbb{I}_{1}^{\text {single }}(j)\right)_{I, I}= \begin{cases}1, & \text { if }|I|=n, j \notin I, \\ 0, & \text { otherwise },\end{cases}
$$

and the matrices $\mathbb{I}_{2}^{\text {single }}(j)$ have non-zero entries only in the lower left corner, i.e.,

$$
\left(\mathbb{I}_{2}^{\text {single }}(j)\right)_{I, j}= \begin{cases}1, & \text { if }|I|=n, j \notin I \\ 0, & \text { otherwise. }\end{cases}
$$

This leads to the following quadratic term:

$$
\begin{aligned}
Q\left(z^{(n)} ; F, \text { Single Split }\right) & =\sum_{j=1}^{N} \operatorname{diag}\left(\mathbb{I}_{1}^{\text {single }}(j) \cdot F\left(z^{(n)}\right)\right) \cdot C^{(n)}(j) \cdot \mathbb{I}_{2}^{\text {single }}(j) \cdot F\left(z^{(n)}\right) \\
& =\sum_{j=1}^{N} \operatorname{diag}\left(F\left(z^{(n)}\right)\right) \cdot \mathbb{I}_{1}^{\text {single }}(j) \cdot C^{(n)}(j) \cdot \mathbb{I}_{2}^{\text {single }}(j) \cdot F\left(z^{(n)}\right) \\
& =\operatorname{diag}\left(F\left(z^{(n)}\right)\right) \cdot\left(\sum_{j=1}^{N} \mathbb{I}_{1}^{\text {single }}(j) \cdot C^{(n)}(j) \cdot \mathbb{I}_{2}^{\text {single }}(j)\right) \cdot F\left(z^{(n)}\right) \\
& =\operatorname{diag}\left(F\left(z^{(n)}\right)\right) \cdot B^{(n)} \cdot F\left(z^{(n)}\right),
\end{aligned}
$$

where the first step follows since $\mathbb{I}_{1}^{\text {single }}(j)$ is diagonal and the matrix $B^{(n)} \in \mathbb{R}^{M \times M}$ is defined by its entries in the lower left corner

$$
\left(B^{(n)}\right)_{I, j}= \begin{cases}\sum_{i \in I} a_{i j}, & \text { if }|I|=n, j \notin I \\ 0, & \text { otherwise. }\end{cases}
$$

Note that in this special case, the quadratic term possesses the exact same structure as in the first-order case.

\section{APPENDIX D: ADJACENCY MATRICES}

We explicitly give the adjacency matrices for the three example networks depicted in Figure 6. The corresponding MATLAB-files are available upon request. 
$\left(\begin{array}{llllllllllllllllllllllllllllllllllllllllllllllllll}0 & 0 & 1 & 0 & 0 & 0 & 0 & 0 & 1 & 0 & 0 & 1 & 0 & 0 & 0 & 0 & 0 & 0 & 0 & 0 & 0 & 1 & 0 & 0 & 0 & 0 & 0 & 0 & 0 & 1 & 0 & 0 & 0 & 0 & 0 & 0 & 0 & 0 & 0 & 0 & 1 & 0 & 0 & 0 & 0 & 0 & 0 & 0 & 1 & 0 \\ 0 & 0 & 0 & 0 & 0 & 0 & 0 & 0\end{array}\right.$ $\left(\begin{array}{llllllllllllllllllllllllllllllllllllllllllllllllllll}0 & 0 & 0 & 0 & 0 & 0 & 0 & 0 & 0 & 0 & 0 & 0 & 0 & 1 & 0 & 0 & 0 & 0 & 0 & 0 & 0 & 0 & 0 & 0 & 0 & 0 & 0 & 1 & 0 & 0 & 0 & 0 & 0 & 0 & 1 & 0 & 1 & 1 & 0 & 0 & 0 & 0 & 1 & 1 & 0 & 0 & 0 & 0 & 0 & 0 \\ 1 & 0 & 0 & 0 & 0 & 0 & 0 & 1 & 0 & 0 & 0 & 0 & 0 & 0 & 0 & 0 & 0 & 0 & 1 & 0 & 1 & 0 & 1 & 0 & 0 & 1 & 0 & 0 & 0 & 0 & 0 & 0 & 0 & 0 & 0 & 0 & 0 & 0 & 0 & 0 & 0 & 0 & 0 & 1 & 0 & 0 & 0 & 0 & 0 & 0\end{array}\right)$ $\begin{array}{llllllllllllllllllllllllllllllllllllllllllllllllll}1 & 0 & 0 & 0 & 0 & 0 & 0 & 1 & 0 & 0 & 0 & 0 & 0 & 0 & 0 & 0 & 0 & 0 & 1 & 0 & 1 & 0 & 1 & 0 & 0 & 1 & 0 & 0 & 0 & 0 & 0 & 0 & 0 & 0 & 0 & 0 & 0 & 0 & 0 & 0 & 0 & 0 & 0 & 1 & 0 & 0 & 0 & 0 & 0 & 0\end{array}$ $\begin{array}{llllllllllllllllllllllllllllllllllllllllllllllllll}0 & 0 & 0 & 0 & 0 & 1 & 0 & 1 & 0 & 1 & 0 & 0 & 0 & 0 & 0 & 0 & 0 & 0 & 0 & 0 & 0 & 0 & 0 & 0 & 0 & 0 & 0 & 0 & 0 & 1 & 0 & 0 & 0 & 0 & 1 & 0 & 1 & 0 & 0 & 0 & 0 & 0 & 0 & 1 & 0 & 0 & 0 & 0 & 0 & 0\end{array}$

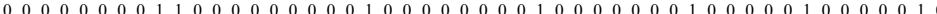

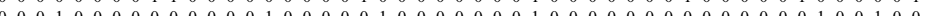

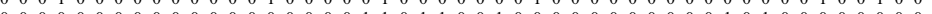

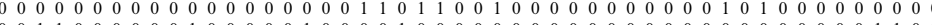
$\begin{array}{lllllllllllllllllllllllllllllllllllllllllllllllllll}0 & 0 & 1 & 1 & 0 & 0 & 0 & 0 & 0 & 0 & 1 & 0 & 0 & 0 & 0 & 0 & 1 & 0 & 0 & 0 & 0 & 1 & 0 & 0 & 0 & 0 & 0 & 0 & 0 & 0 & 0 & 0 & 0 & 0 & 0 & 0 & 0 & 0 & 0 & 0 & 0 & 0 & 0 & 0 & 0 & 0 & 1 & 1 & 0 & 0\end{array}$ $\begin{array}{lllllllllllllllllllllllllllllllllllllllllllllllllll}1 & 0 & 0 & 0 & 1 & 0 & 0 & 0 & 0 & 0 & 0 & 0 & 0 & 0 & 1 & 1 & 0 & 0 & 1 & 0 & 0 & 0 & 0 & 0 & 0 & 0 & 0 & 0 & 0 & 0 & 1 & 0 & 1 & 0 & 0 & 0 & 0 & 0 & 0 & 0 & 0 & 0 & 0 & 0 & 0 & 0 & 0 & 0 & 0 & 0\end{array}$ $\begin{array}{llllllllllllllllllllllllllllllllllllllllllllllllll}0 & 0 & 0 & 1 & 1 & 0 & 0 & 0 & 0 & 0 & 0 & 0 & 0 & 0 & 0 & 1 & 0 & 0 & 0 & 0 & 0 & 0 & 0 & 0 & 1 & 1 & 0 & 1 & 0 & 0 & 0 & 0 & 0 & 1 & 0 & 0 & 0 & 0 & 0 & 0 & 0 & 0 & 0 & 0 & 0 & 0 & 0 & 0 & 0 & 0\end{array}$ $\begin{array}{lllllllllllllllllllllllllllllllllllllllllllllllllll}0 & 0 & 0 & 0 & 0 & 0 & 0 & 1 & 0 & 0 & 0 & 0 & 0 & 0 & 1 & 0 & 0 & 0 & 0 & 1 & 0 & 0 & 0 & 0 & 1 & 1 & 0 & 0 & 0 & 0 & 1 & 0 & 0 & 0 & 0 & 0 & 0 & 0 & 0 & 0 & 0 & 0 & 0 & 0 & 0 & 0 & 0 & 1 & 0 & 0\end{array}$ $\begin{array}{lllllllllllllllllllllllllllllllllllllllllllllllllll}1 & 0 & 0 & 0 & 0 & 0 & 0 & 0 & 0 & 0 & 0 & 0 & 0 & 0 & 0 & 0 & 0 & 0 & 0 & 0 & 0 & 1 & 0 & 0 & 0 & 1 & 0 & 0 & 0 & 0 & 1 & 0 & 0 & 1 & 0 & 0 & 0 & 0 & 0 & 0 & 1 & 1 & 0 & 0 & 0 & 0 & 0 & 0 & 0 & 0\end{array}$

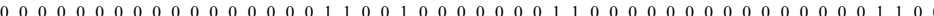

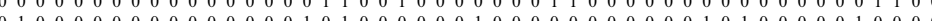

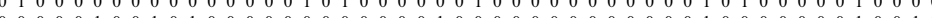
$\begin{array}{llllllllllllllllllllllllllllllllllllllllllllllllllllllll}0 & 0 & 0 & 0 & 0 & 1 & 0 & 0 & 1 & 0 & 1 & 0 & 0 & 0 & 0 & 0 & 0 & 0 & 0 & 0 & 0 & 0 & 0 & 1 & 0 & 0 & 0 & 0 & 0 & 0 & 0 & 0 & 0 & 0 & 0 & 0 & 0 & 1 & 0 & 0 & 0 & 0 & 0 & 0 & 0 & 1 & 0 & 0 & 1 & 0\end{array}$ $\begin{array}{llllllllllllllllllllllllllllllllllllllllllllllllll}0 & 0 & 0 & 0 & 0 & 0 & 0 & 0 & 1 & 1 & 0 & 0 & 0 & 0 & 0 & 0 & 0 & 0 & 0 & 0 & 0 & 0 & 0 & 1 & 1 & 0 & 0 & 1 & 0 & 0 & 0 & 0 & 1 & 0 & 0 & 0 & 0 & 0 & 0 & 0 & 0 & 0 & 1 & 0 & 0 & 0 & 0 & 0 & 0 & 0\end{array}$ $\begin{array}{llllllllllllllllllllllllllllllllllllllllllllllllll}0 & 0 & 0 & 0 & 0 & 0 & 0 & 1 & 0 & 0 & 0 & 0 & 0 & 1 & 0 & 0 & 0 & 0 & 0 & 0 & 0 & 0 & 0 & 0 & 0 & 0 & 0 & 1 & 0 & 0 & 0 & 0 & 0 & 0 & 0 & 0 & 0 & 0 & 1 & 0 & 0 & 1 & 0 & 0 & 0 & 0 & 1 & 0 & 0 & 1\end{array}$ $\begin{array}{llllllllllllllllllllllllllllllllllllllllllllllllll}0 & 0 & 0 & 0 & 0 & 0 & 0 & 0 & 0 & 0 & 0 & 0 & 1 & 0 & 0 & 0 & 0 & 0 & 0 & 0 & 0 & 0 & 0 & 1 & 1 & 0 & 0 & 0 & 1 & 0 & 0 & 0 & 0 & 0 & 0 & 1 & 0 & 0 & 0 & 0 & 0 & 0 & 0 & 0 & 1 & 0 & 0 & 1 & 0 & 0\end{array}$ $\begin{array}{llllllllllllllllllllllllllllllllllllllllllllllllll}0 & 0 & 1 & 0 & 0 & 0 & 0 & 0 & 1 & 0 & 0 & 0 & 1 & 1 & 0 & 0 & 0 & 0 & 0 & 0 & 0 & 0 & 0 & 0 & 0 & 0 & 0 & 0 & 1 & 0 & 0 & 0 & 0 & 0 & 0 & 0 & 0 & 0 & 0 & 1 & 0 & 0 & 0 & 0 & 1 & 0 & 0 & 0 & 0 & 0\end{array}$ $\begin{array}{llllllllllllllllllllllllllllllllllllllllllllllllll}0 & 0 & 0 & 0 & 1 & 0 & 1 & 0 & 0 & 0 & 1 & 0 & 0 & 0 & 0 & 0 & 0 & 0 & 0 & 0 & 0 & 0 & 0 & 0 & 0 & 0 & 0 & 0 & 0 & 0 & 0 & 0 & 0 & 0 & 0 & 1 & 1 & 0 & 0 & 0 & 0 & 1 & 0 & 0 & 1 & 0 & 0 & 0 & 0 & 0\end{array}$ $\begin{array}{llllllllllllllllllllllllllllllllllllllllllllllllll}0 & 0 & 1 & 0 & 0 & 1 & 1 & 0 & 0 & 0 & 0 & 0 & 0 & 0 & 0 & 0 & 0 & 0 & 0 & 0 & 0 & 0 & 0 & 0 & 0 & 0 & 1 & 0 & 0 & 0 & 0 & 0 & 0 & 0 & 1 & 0 & 0 & 0 & 0 & 1 & 0 & 0 & 0 & 0 & 0 & 1 & 0 & 0 & 0 & 0\end{array}$ $\begin{array}{lllllllllllllllllllllllllllllllllllllllllllllllllll}1 & 0 & 0 & 0 & 0 & 0 & 0 & 1 & 0 & 0 & 0 & 1 & 1 & 0 & 0 & 0 & 0 & 0 & 0 & 0 & 0 & 0 & 0 & 0 & 0 & 0 & 1 & 0 & 0 & 0 & 1 & 0 & 0 & 1 & 0 & 0 & 0 & 0 & 0 & 0 & 0 & 0 & 0 & 0 & 0 & 0 & 0 & 0 & 0 & 0\end{array}$ $\begin{array}{lllllllllllllllllllllllllllllllllllllllllllllllllll}0 & 0 & 1 & 0 & 0 & 0 & 1 & 0 & 0 & 0 & 0 & 0 & 0 & 0 & 0 & 0 & 0 & 0 & 0 & 0 & 0 & 0 & 0 & 1 & 0 & 0 & 0 & 0 & 0 & 0 & 0 & 1 & 0 & 0 & 0 & 1 & 0 & 1 & 0 & 1 & 0 & 0 & 0 & 0 & 0 & 0 & 0 & 0 & 0 & 0\end{array}$ $\begin{array}{lllllllllllllllllllllllllllllllllllllllllllllllllllll}0 & 0 & 0 & 0 & 0 & 0 & 1 & 0 & 0 & 0 & 0 & 0 & 0 & 0 & 1 & 1 & 0 & 1 & 0 & 0 & 0 & 0 & 1 & 0 & 0 & 0 & 0 & 0 & 0 & 0 & 0 & 0 & 1 & 0 & 0 & 0 & 0 & 0 & 0 & 0 & 0 & 0 & 0 & 1 & 0 & 0 & 0 & 0 & 0 & 0\end{array}$ $\begin{array}{lllllllllllllllllllllllllllllllllllllllllllllllllllllll}0 & 0 & 0 & 0 & 0 & 0 & 0 & 0 & 0 & 1 & 1 & 0 & 0 & 0 & 0 & 1 & 0 & 1 & 0 & 0 & 0 & 0 & 0 & 0 & 0 & 0 & 0 & 0 & 0 & 0 & 1 & 0 & 0 & 0 & 0 & 0 & 0 & 0 & 0 & 0 & 0 & 0 & 0 & 0 & 0 & 1 & 0 & 1 & 0 & 0\end{array}$ $\begin{array}{lllllllllllllllllllllllllllllllllllllllllllllllllll}0 & 0 & 1 & 0 & 0 & 0 & 0 & 0 & 0 & 1 & 1 & 1 & 0 & 1 & 0 & 0 & 0 & 0 & 0 & 0 & 0 & 0 & 0 & 0 & 0 & 0 & 0 & 0 & 0 & 0 & 0 & 0 & 1 & 0 & 0 & 0 & 0 & 0 & 0 & 1 & 0 & 0 & 0 & 0 & 0 & 0 & 0 & 0 & 0 & 0\end{array}$ $\begin{array}{llllllllllllllllllllllllllllllllllllllllllllllllll}0 & 0 & 0 & 0 & 0 & 0 & 1 & 0 & 0 & 0 & 0 & 0 & 0 & 0 & 0 & 0 & 0 & 0 & 0 & 0 & 1 & 1 & 0 & 0 & 0 & 0 & 0 & 1 & 0 & 0 & 0 & 0 & 0 & 0 & 0 & 1 & 0 & 1 & 0 & 0 & 0 & 1 & 0 & 0 & 0 & 0 & 0 & 0 & 0 & 0\end{array}$ $\begin{array}{llllllllllllllllllllllllllllllllllllllllllllllllll}0 & 1 & 0 & 0 & 0 & 0 & 0 & 0 & 0 & 1 & 0 & 0 & 0 & 0 & 0 & 1 & 1 & 0 & 0 & 0 & 0 & 0 & 0 & 0 & 0 & 0 & 1 & 0 & 0 & 0 & 0 & 0 & 0 & 0 & 0 & 0 & 0 & 0 & 0 & 0 & 1 & 0 & 1 & 0 & 0 & 0 & 0 & 0 & 0 & 0\end{array}$ $\begin{array}{llllllllllllllllllllllllllllllllllllllllllllllllll}0 & 0 & 0 & 0 & 1 & 1 & 0 & 0 & 0 & 0 & 0 & 0 & 0 & 0 & 0 & 0 & 0 & 1 & 1 & 0 & 0 & 0 & 0 & 0 & 0 & 0 & 0 & 0 & 0 & 0 & 1 & 0 & 0 & 0 & 0 & 0 & 0 & 0 & 0 & 0 & 1 & 0 & 0 & 0 & 0 & 1 & 0 & 0 & 0 & 0\end{array}$ $\begin{array}{llllllllllllllllllllllllllllllllllllllllllllllllllll}1 & 0 & 0 & 1 & 0 & 0 & 0 & 0 & 0 & 0 & 0 & 0 & 1 & 0 & 0 & 0 & 0 & 0 & 0 & 0 & 0 & 0 & 0 & 0 & 0 & 0 & 0 & 0 & 0 & 0 & 0 & 0 & 0 & 0 & 0 & 0 & 0 & 0 & 0 & 0 & 0 & 0 & 0 & 1 & 1 & 0 & 1 & 0 & 1 & 0\end{array}$

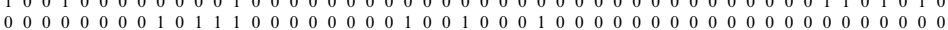
$\begin{array}{llllllllllllllllllllllllllllllllllllllllllllllllllll}0 & 0 & 0 & 0 & 0 & 0 & 0 & 0 & 0 & 0 & 0 & 0 & 0 & 0 & 0 & 0 & 0 & 0 & 0 & 0 & 0 & 0 & 1 & 0 & 0 & 0 & 0 & 0 & 0 & 0 & 0 & 0 & 0 & 1 & 0 & 0 & 0 & 0 & 1 & 1 & 0 & 0 & 0 & 0 & 0 & 0 & 1 & 0 & 1 & 1\end{array}$ $\begin{array}{lllllllllllllllllllllllllllllllllllllllllllllllllll}0 & 0 & 0 & 0 & 0 & 0 & 0 & 0 & 1 & 0 & 0 & 0 & 0 & 0 & 0 & 1 & 0 & 0 & 0 & 0 & 0 & 0 & 0 & 1 & 0 & 1 & 0 & 0 & 0 & 0 & 0 & 0 & 0 & 1 & 0 & 1 & 0 & 0 & 0 & 0 & 0 & 0 & 0 & 0 & 1 & 0 & 0 & 0 & 0 & 0\end{array}$ $\begin{array}{llllllllllllllllllllllllllllllllllllllllllllllllllll}0 & 0 & 0 & 0 & 0 & 0 & 0 & 0 & 0 & 1 & 0 & 1 & 0 & 0 & 0 & 0 & 0 & 0 & 0 & 0 & 0 & 1 & 0 & 0 & 0 & 0 & 0 & 0 & 0 & 0 & 0 & 1 & 1 & 0 & 1 & 0 & 0 & 0 & 0 & 0 & 0 & 0 & 0 & 0 & 0 & 0 & 0 & 0 & 0 & 1\end{array}$ $\begin{array}{llllllllllllllllllllllllllllllllllllllllllllllllll}0 & 1 & 0 & 1 & 0 & 0 & 0 & 0 & 0 & 0 & 0 & 0 & 0 & 0 & 0 & 0 & 0 & 0 & 0 & 0 & 1 & 0 & 0 & 0 & 0 & 0 & 0 & 0 & 0 & 0 & 0 & 0 & 0 & 1 & 0 & 0 & 0 & 0 & 1 & 0 & 0 & 0 & 1 & 0 & 0 & 1 & 0 & 0 & 0 & 0\end{array}$ $\begin{array}{lllllllllllllllllllllllllllllllllllllllllllllllllll}0 & 0 & 0 & 0 & 0 & 0 & 0 & 0 & 0 & 0 & 0 & 0 & 0 & 0 & 0 & 0 & 0 & 1 & 0 & 1 & 0 & 0 & 1 & 0 & 0 & 0 & 1 & 0 & 0 & 0 & 0 & 0 & 1 & 0 & 0 & 0 & 1 & 0 & 0 & 0 & 0 & 1 & 0 & 0 & 0 & 0 & 0 & 0 & 0 & 0\end{array}$ $\begin{array}{llllllllllllllllllllllllllllllllllllllllllllllllll}0 & 1 & 0 & 1 & 1 & 0 & 0 & 0 & 0 & 0 & 0 & 0 & 0 & 0 & 0 & 0 & 0 & 0 & 0 & 1 & 0 & 0 & 0 & 0 & 0 & 0 & 0 & 0 & 0 & 0 & 0 & 0 & 0 & 0 & 0 & 1 & 0 & 0 & 0 & 0 & 0 & 1 & 0 & 0 & 0 & 0 & 0 & 0 & 1 & 0\end{array}$ $\begin{array}{llllllllllllllllllllllllllllllllllllllllllllllllllll}0 & 1 & 0 & 0 & 0 & 0 & 0 & 0 & 0 & 0 & 0 & 0 & 0 & 1 & 1 & 0 & 0 & 0 & 0 & 0 & 0 & 0 & 1 & 0 & 0 & 0 & 1 & 0 & 0 & 0 & 0 & 0 & 0 & 0 & 0 & 0 & 0 & 0 & 0 & 0 & 1 & 0 & 1 & 0 & 0 & 0 & 0 & 0 & 0 & 0\end{array}$ $\begin{array}{llllllllllllllllllllllllllllllllllllllllllllllllllll}0 & 0 & 0 & 0 & 0 & 0 & 1 & 0 & 0 & 0 & 0 & 0 & 0 & 0 & 0 & 0 & 1 & 0 & 0 & 0 & 0 & 0 & 0 & 0 & 0 & 0 & 0 & 0 & 0 & 0 & 0 & 1 & 0 & 0 & 1 & 0 & 0 & 0 & 0 & 0 & 0 & 0 & 0 & 0 & 1 & 0 & 0 & 1 & 0 & 1\end{array}$ $\begin{array}{lllllllllllllllllllllllllllllllllllllllllllllllllllllllllll}0 & 0 & 0 & 0 & 0 & 0 & 0 & 0 & 0 & 0 & 0 & 0 & 0 & 1 & 0 & 0 & 0 & 0 & 1 & 0 & 1 & 0 & 1 & 0 & 0 & 1 & 0 & 0 & 0 & 0 & 0 & 1 & 0 & 0 & 0 & 0 & 0 & 0 & 0 & 0 & 0 & 0 & 1 & 0 & 0 & 0 & 0 & 0 & 0 & 0\end{array}$ $\begin{array}{llllllllllllllllllllllllllllllllllllllllllllllllll}1 & 0 & 0 & 0 & 0 & 0 & 1 & 0 & 0 & 0 & 0 & 1 & 0 & 0 & 0 & 0 & 0 & 0 & 0 & 0 & 0 & 0 & 0 & 0 & 0 & 0 & 0 & 1 & 1 & 0 & 0 & 0 & 0 & 0 & 0 & 0 & 0 & 1 & 0 & 0 & 0 & 0 & 0 & 0 & 0 & 0 & 0 & 0 & 0 & 1\end{array}$ $\begin{array}{lllllllllllllllllllllllllllllllllllllllllllllllllll}0 & 0 & 0 & 0 & 0 & 0 & 0 & 0 & 0 & 0 & 0 & 1 & 0 & 0 & 0 & 0 & 1 & 0 & 0 & 1 & 0 & 0 & 0 & 0 & 0 & 0 & 1 & 0 & 0 & 0 & 0 & 0 & 0 & 0 & 0 & 1 & 1 & 0 & 0 & 0 & 0 & 0 & 0 & 0 & 0 & 0 & 0 & 1 & 0 & 0\end{array}$ $\begin{array}{llllllllllllllllllllllllllllllllllllllllllllllllll}0 & 1 & 0 & 0 & 1 & 0 & 0 & 0 & 0 & 0 & 0 & 0 & 0 & 0 & 0 & 1 & 0 & 0 & 0 & 0 & 0 & 0 & 0 & 0 & 0 & 0 & 0 & 1 & 0 & 0 & 0 & 0 & 0 & 0 & 1 & 0 & 0 & 1 & 0 & 1 & 0 & 0 & 0 & 0 & 0 & 0 & 0 & 0 & 0 & 0\end{array}$ $\begin{array}{lllllllllllllllllllllllllllllllllllllllllllllllllll}0 & 1 & 1 & 1 & 0 & 1 & 0 & 0 & 0 & 0 & 0 & 0 & 0 & 0 & 0 & 0 & 0 & 0 & 0 & 0 & 0 & 0 & 0 & 1 & 0 & 0 & 0 & 0 & 0 & 1 & 0 & 0 & 0 & 0 & 0 & 0 & 0 & 0 & 0 & 0 & 0 & 0 & 0 & 0 & 0 & 0 & 0 & 0 & 1 & 0\end{array}$

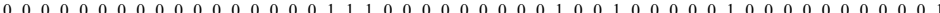

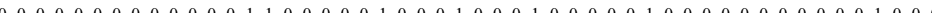
0

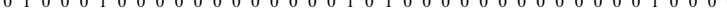
$\begin{array}{lllllllllllllllllllllllllllllllllllllllllllllllllll}0 & 0 & 0 & 0 & 0 & 0 & 0 & 1 & 0 & 0 & 1 & 0 & 1 & 0 & 0 & 0 & 0 & 1 & 0 & 0 & 0 & 0 & 0 & 0 & 1 & 0 & 0 & 0 & 0 & 0 & 0 & 0 & 0 & 0 & 0 & 0 & 0 & 0 & 1 & 0 & 0 & 1 & 0 & 0 & 0 & 0 & 0 & 0 & 0 & 0\end{array}$ $\begin{array}{lllllllllllllllllllllllllllllllllllllllllllllllll}1 & 0 & 0 & 0 & 1 & 0 & 0 & 0 & 0 & 0 & 0 & 0 & 0 & 0 & 1 & 0 & 0 & 0 & 0 & 0 & 0 & 0 & 0 & 0 & 0 & 0 & 0 & 0 & 0 & 1 & 0 & 1 & 0 & 0 & 0 & 0 & 1 & 0 & 0 & 0 & 0 & 0 & 0 & 1 & 0 & 0 & 0 & 0 & 0\end{array}$ $\left(\begin{array}{lllllllllllllllllllllllllllllllllllllllllllllllllllllllllllllllllllll}0 & 0 & 0 & 0 & 0 & 1 & 0 & 0 & 0 & 0 & 0 & 0 & 0 & 0 & 0 & 0 & 1 & 0 & 0 & 0 & 0 & 0 & 0 & 0 & 0 & 0 & 0 & 0 & 0 & 0 & 0 & 1 & 0 & 1 & 0 & 0 & 0 & 0 & 1 & 0 & 1 & 0 & 0 & 0 & 1 & 0 & 0 & 0 & 0 & 0\end{array}\right)$ 
$\left(\begin{array}{llllllllllllllllllllllllllllllllllllllllllllllllll}0 & 0 & 1 & 0 & 1 & 1 & 0 & 1 & 1 & 1 & 1 & 0 & 0 & 0 & 0 & 0 & 0 & 0 & 0 & 0 & 0 & 0 & 0 & 0 & 0 & 0 & 0 & 0 & 0 & 0 & 0 & 0 & 0 & 0 & 0 & 0 & 0 & 0 & 0 & 0 & 0 & 0 & 0 & 0 & 0 & 0 & 0 & 0 & 0 & 0\end{array}\right.$ $\begin{array}{llllllllllllllllllllllllllllllllllllllllllllllllll}0 & 0 & 1 & 1 & 1 & 0 & 1 & 1 & 0 & 1 & 0 & 0 & 0 & 0 & 0 & 0 & 0 & 0 & 0 & 0 & 0 & 0 & 0 & 0 & 0 & 0 & 0 & 0 & 0 & 0 & 0 & 0 & 0 & 0 & 0 & 0 & 0 & 0 & 0 & 0 & 0 & 1 & 0 & 0 & 0 & 0 & 0 & 0 & 0 & 0 \\ 1 & 1 & 0 & 1 & 0 & 1 & 1 & 1 & 1 & 0 & 0 & 0 & 0 & 0 & 0 & 0 & 0 & 0 & 0 & 0 & 0 & 0 & 0 & 0 & 0 & 0 & 0 & 0 & 0 & 0 & 0 & 0 & 0 & 0 & 0 & 0 & 0 & 0 & 0 & 0 & 0 & 0 & 0 & 0 & 0 & 0 & 0 & 0 & 0 & 0\end{array}$ $\begin{array}{lllllllllllllllllllllllllllllllllllllllllllllllllll}1 & 1 & 0 & 1 & 0 & 1 & 1 & 1 & 1 & 0 & 0 & 0 & 0 & 0 & 0 & 0 & 0 & 0 & 0 & 0 & 0 & 0 & 0 & 0 & 0 & 0 & 0 & 0 & 0 & 0 & 0 & 0 & 0 & 0 & 0 & 0 & 0 & 0 & 0 & 0 & 0 & 0 & 0 & 0 & 0 & 0 & 0 & 0 & 0 & 0\end{array}$

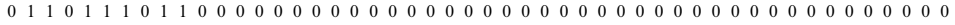
$\begin{array}{lllllllllllllllllllllllllllllllllllllllllllllllllll}1 & 1 & 0 & 1 & 0 & 1 & 1 & 1 & 1 & 0 & 0 & 0 & 0 & 0 & 0 & 0 & 0 & 0 & 0 & 0 & 0 & 0 & 0 & 0 & 0 & 0 & 0 & 0 & 0 & 0 & 0 & 0 & 0 & 0 & 0 & 0 & 0 & 0 & 0 & 0 & 0 & 0 & 0 & 0 & 0 & 0 & 0 & 0 & 0 & 0\end{array}$

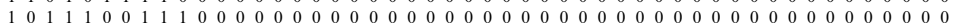
$\begin{array}{lllllllllllllllllllllllllllllllllllllllllllllllllll}0 & 1 & 1 & 1 & 1 & 0 & 0 & 1 & 1 & 1 & 0 & 0 & 0 & 0 & 0 & 0 & 0 & 0 & 0 & 0 & 0 & 0 & 0 & 0 & 0 & 0 & 0 & 0 & 0 & 0 & 0 & 0 & 0 & 0 & 0 & 0 & 0 & 0 & 0 & 0 & 0 & 0 & 0 & 0 & 0 & 0 & 0 & 0 & 0 & 0\end{array}$

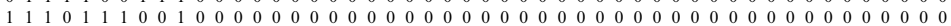

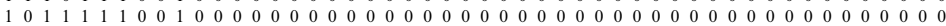
$\begin{array}{llllllllllllllllllllllllllllllllllllllllllllllllll}1 & 1 & 0 & 1 & 0 & 1 & 1 & 1 & 1 & 0 & 0 & 0 & 0 & 0 & 0 & 0 & 0 & 0 & 0 & 0 & 0 & 0 & 0 & 0 & 0 & 0 & 0 & 0 & 0 & 0 & 0 & 0 & 0 & 0 & 0 & 0 & 0 & 0 & 0 & 0 & 0 & 0 & 0 & 0 & 0 & 0 & 0 & 0 & 0 & 0\end{array}$ $\begin{array}{lllllllllllllllllllllllllllllllllllllllllllllllllll}1 & 0 & 0 & 0 & 0 & 0 & 0 & 0 & 0 & 0 & 0 & 0 & 0 & 1 & 1 & 1 & 1 & 1 & 0 & 1 & 0 & 0 & 0 & 0 & 0 & 0 & 0 & 0 & 0 & 0 & 0 & 0 & 0 & 0 & 0 & 0 & 0 & 0 & 0 & 0 & 0 & 0 & 0 & 0 & 0 & 0 & 0 & 0 & 0 & 0\end{array}$

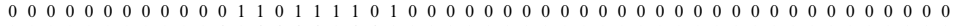

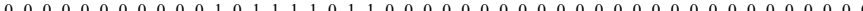

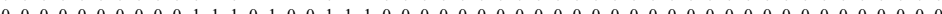

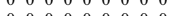
$\begin{array}{lllllllllllllllllllllllllllllllllllllllllllllllll}0 & 0 & 0 & 0 & 0 & 0 & 0 & 0 & 0 & 0 & 1 & 1 & 1 & 0 & 0 & 0 & 1 & 1 & 1 & 1 & 0 & 0 & 0 & 0 & 0 & 0 & 0 & 0 & 0 & 0 & 0 & 0 & 0 & 0 & 0 & 0 & 0 & 0 & 0 & 0 & 0 & 0 & 0 & 0 & 0 & 0 & 0 & 0 & 0\end{array}$

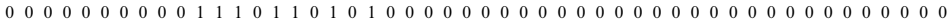
$\begin{array}{lllllllllllllllllllllllllllllllllllllllllllllllllll}0 & 0 & 0 & 0 & 0 & 0 & 0 & 0 & 0 & 0 & 1 & 1 & 0 & 1 & 1 & 1 & 1 & 0 & 1 & 0 & 0 & 0 & 0 & 0 & 0 & 0 & 0 & 0 & 0 & 0 & 0 & 0 & 0 & 0 & 0 & 0 & 0 & 0 & 0 & 0 & 0 & 0 & 0 & 0 & 0 & 0 & 0 & 0 & 0 & 0\end{array}$ $\begin{array}{lllllllllllllllllllllllllllllllllllllllllllllllllllll}0 & 0 & 0 & 0 & 0 & 0 & 0 & 0 & 0 & 0 & 0 & 1 & 1 & 1 & 1 & 1 & 0 & 1 & 0 & 1 & 0 & 0 & 0 & 0 & 0 & 0 & 0 & 0 & 0 & 0 & 0 & 0 & 0 & 0 & 0 & 0 & 0 & 0 & 0 & 0 & 0 & 0 & 0 & 0 & 0 & 0 & 0 & 0 & 0 & 0\end{array}$

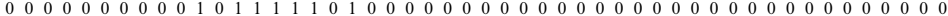

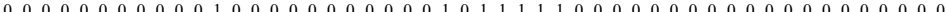

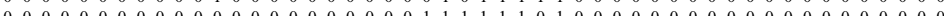
$\begin{array}{llllllllllllllllllllllllllllllllllllllllllllllllll}0 & 0 & 0 & 0 & 0 & 0 & 0 & 0 & 0 & 0 & 0 & 0 & 0 & 0 & 0 & 0 & 0 & 0 & 0 & 0 & 1 & 1 & 1 & 0 & 1 & 1 & 0 & 1 & 0 & 1 & 0 & 0 & 0 & 0 & 0 & 0 & 0 & 0 & 0 & 0 & 0 & 0 & 0 & 0 & 0 & 0 & 0 & 0 & 0 & 0\end{array}$

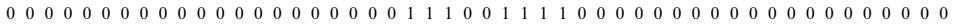
$\begin{array}{llllllllllllllllllllllllllllllllllllllllllllllllll}0 & 0 & 0 & 0 & 0 & 0 & 0 & 0 & 0 & 0 & 0 & 0 & 0 & 0 & 0 & 0 & 0 & 0 & 0 & 0 & 1 & 1 & 1 & 1 & 0 & 0 & 1 & 1 & 1 & 0 & 0 & 0 & 0 & 0 & 0 & 0 & 0 & 0 & 0 & 0 & 0 & 0 & 0 & 0 & 0 & 0 & 0 & 0 & 0 & 0\end{array}$ $\begin{array}{lllllllllllllllllllllllllllllllllllllllllllllllllll}0 & 0 & 0 & 0 & 0 & 0 & 0 & 0 & 0 & 0 & 0 & 0 & 0 & 0 & 0 & 0 & 0 & 0 & 0 & 0 & 1 & 1 & 1 & 0 & 1 & 1 & 0 & 0 & 1 & 1 & 0 & 0 & 0 & 0 & 0 & 0 & 0 & 0 & 0 & 0 & 0 & 0 & 0 & 0 & 0 & 0 & 0 & 0 & 0 & 0\end{array}$

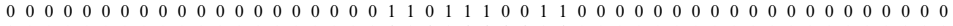
$\begin{array}{llllllllllllllllllllllllllllllllllllllllllllllllll}0 & 0 & 0 & 0 & 0 & 0 & 0 & 0 & 0 & 0 & 0 & 0 & 0 & 0 & 0 & 0 & 0 & 0 & 0 & 0 & 1 & 0 & 1 & 0 & 1 & 1 & 1 & 1 & 0 & 1 & 0 & 0 & 0 & 0 & 0 & 0 & 0 & 0 & 0 & 0 & 0 & 0 & 0 & 0 & 0 & 0 & 0 & 0 & 0 & 0\end{array}$ $\begin{array}{lllllllllllllllllllllllllllllllllllllllllllllllllllllll}0 & 0 & 0 & 0 & 0 & 0 & 0 & 0 & 0 & 0 & 0 & 0 & 0 & 0 & 0 & 0 & 0 & 0 & 0 & 0 & 1 & 1 & 0 & 1 & 1 & 0 & 1 & 1 & 1 & 0 & 0 & 0 & 0 & 0 & 0 & 0 & 0 & 0 & 0 & 0 & 0 & 0 & 0 & 0 & 0 & 0 & 0 & 0 & 0 & 0\end{array}$ $\begin{array}{llllllllllllllllllllllllllllllllllllllllllllllllllllll}0 & 0 & 0 & 0 & 0 & 0 & 0 & 0 & 0 & 0 & 0 & 0 & 0 & 0 & 0 & 0 & 0 & 0 & 0 & 0 & 0 & 0 & 1 & 0 & 0 & 0 & 0 & 0 & 0 & 0 & 0 & 0 & 0 & 0 & 1 & 1 & 1 & 1 & 1 & 1 & 0 & 0 & 0 & 0 & 0 & 0 & 0 & 0 & 0 & 0\end{array}$ $\begin{array}{lllllllllllllllllllllllllllllllllllllllllllllllllllllll}0 & 0 & 0 & 0 & 0 & 0 & 0 & 0 & 0 & 0 & 0 & 0 & 0 & 0 & 0 & 0 & 0 & 0 & 0 & 0 & 0 & 0 & 0 & 0 & 0 & 0 & 0 & 0 & 0 & 0 & 0 & 0 & 1 & 1 & 1 & 1 & 1 & 0 & 1 & 1 & 0 & 0 & 0 & 0 & 0 & 0 & 0 & 0 & 0 & 0\end{array}$ $\begin{array}{llllllllllllllllllllllllllllllllllllllllllllllllll}0 & 0 & 0 & 0 & 0 & 0 & 0 & 0 & 0 & 0 & 0 & 0 & 0 & 0 & 0 & 0 & 0 & 0 & 0 & 0 & 0 & 0 & 0 & 0 & 0 & 0 & 0 & 0 & 0 & 0 & 0 & 1 & 0 & 1 & 1 & 0 & 1 & 0 & 1 & 1 & 1 & 0 & 0 & 0 & 0 & 0 & 0 & 0 & 0 & 0\end{array}$ $\begin{array}{lllllllllllllllllllllllllllllllllllllllllllllllllll}0 & 0 & 0 & 0 & 0 & 0 & 0 & 0 & 0 & 0 & 0 & 0 & 0 & 0 & 0 & 0 & 0 & 0 & 0 & 0 & 0 & 0 & 0 & 0 & 0 & 0 & 0 & 0 & 0 & 0 & 0 & 1 & 1 & 0 & 1 & 1 & 1 & 1 & 1 & 0 & 0 & 0 & 0 & 0 & 0 & 0 & 0 & 0 & 0 & 0\end{array}$ $\begin{array}{lllllllllllllllllllllllllllllllllllllllllllllllllll}0 & 0 & 0 & 0 & 0 & 0 & 0 & 0 & 0 & 0 & 0 & 0 & 0 & 0 & 0 & 0 & 0 & 0 & 0 & 0 & 0 & 0 & 0 & 0 & 0 & 0 & 0 & 0 & 0 & 0 & 1 & 1 & 1 & 1 & 0 & 1 & 0 & 1 & 1 & 0 & 0 & 0 & 0 & 0 & 0 & 0 & 0 & 0 & 0 & 0\end{array}$ $\begin{array}{lllllllllllllllllllllllllllllllllllllllllllllllllll}0 & 0 & 0 & 0 & 0 & 0 & 0 & 0 & 0 & 0 & 0 & 0 & 0 & 0 & 0 & 0 & 0 & 0 & 0 & 0 & 0 & 0 & 0 & 0 & 0 & 0 & 0 & 0 & 0 & 0 & 1 & 1 & 0 & 1 & 1 & 0 & 1 & 1 & 0 & 1 & 0 & 0 & 0 & 0 & 0 & 0 & 0 & 0 & 0 & 0\end{array}$ $\begin{array}{lllllllllllllllllllllllllllllllllllllllllllllllllll}0 & 0 & 0 & 0 & 0 & 0 & 0 & 0 & 0 & 0 & 0 & 0 & 0 & 0 & 0 & 0 & 0 & 0 & 0 & 0 & 0 & 0 & 0 & 0 & 0 & 0 & 0 & 0 & 0 & 0 & 1 & 1 & 1 & 1 & 0 & 1 & 0 & 1 & 0 & 1 & 0 & 0 & 0 & 0 & 0 & 0 & 0 & 0 & 0 & 0\end{array}$ $\begin{array}{llllllllllllllllllllllllllllllllllllllllllllllllll}0 & 0 & 0 & 0 & 0 & 0 & 0 & 0 & 0 & 0 & 0 & 0 & 0 & 0 & 0 & 0 & 0 & 0 & 0 & 0 & 0 & 0 & 0 & 0 & 0 & 0 & 0 & 0 & 0 & 0 & 1 & 0 & 0 & 1 & 1 & 1 & 1 & 0 & 1 & 1 & 0 & 0 & 0 & 0 & 0 & 0 & 0 & 0 & 0 & 0\end{array}$

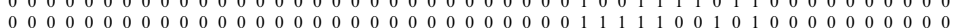

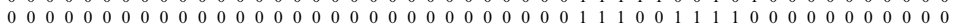
$\begin{array}{llllllllllllllllllllllllllllllllllllllllllllllllll}0 & 0 & 0 & 0 & 0 & 0 & 0 & 0 & 0 & 0 & 0 & 0 & 0 & 0 & 0 & 0 & 0 & 0 & 0 & 0 & 0 & 0 & 0 & 0 & 0 & 0 & 0 & 0 & 0 & 0 & 0 & 0 & 1 & 0 & 0 & 0 & 0 & 0 & 0 & 0 & 0 & 0 & 0 & 1 & 1 & 1 & 1 & 1 & 1 & 0\end{array}$

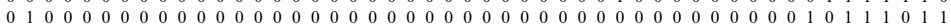
$\begin{array}{lllllllllllllllllllllllllllllllllllllllllllllllllll}0 & 0 & 0 & 0 & 0 & 0 & 0 & 0 & 0 & 0 & 0 & 0 & 0 & 0 & 0 & 0 & 0 & 0 & 0 & 0 & 0 & 0 & 0 & 0 & 0 & 0 & 0 & 0 & 0 & 0 & 0 & 0 & 0 & 0 & 0 & 0 & 0 & 0 & 0 & 0 & 0 & 1 & 0 & 1 & 1 & 1 & 1 & 0 & 1 & 1\end{array}$ $\begin{array}{lllllllllllllllllllllllllllllllllllllllllllllllllll}0 & 0 & 0 & 0 & 0 & 0 & 0 & 0 & 0 & 0 & 0 & 0 & 0 & 0 & 0 & 0 & 0 & 0 & 0 & 0 & 0 & 0 & 0 & 0 & 0 & 0 & 0 & 0 & 0 & 0 & 0 & 0 & 0 & 0 & 0 & 0 & 0 & 0 & 0 & 0 & 1 & 0 & 1 & 0 & 1 & 1 & 0 & 1 & 1 & 1\end{array}$ $\begin{array}{llllllllllllllllllllllllllllllllllllllllllllllllll}0 & 0 & 0 & 0 & 0 & 0 & 0 & 0 & 0 & 0 & 0 & 0 & 0 & 0 & 0 & 0 & 0 & 0 & 0 & 0 & 0 & 0 & 0 & 0 & 0 & 0 & 0 & 0 & 0 & 0 & 0 & 0 & 0 & 0 & 0 & 0 & 0 & 0 & 0 & 0 & 1 & 1 & 1 & 1 & 0 & 0 & 1 & 1 & 0 & 1\end{array}$ $\begin{array}{llllllllllllllllllllllllllllllllllllllllllllllllll}0 & 0 & 0 & 0 & 0 & 0 & 0 & 0 & 0 & 0 & 0 & 0 & 0 & 0 & 0 & 0 & 0 & 0 & 0 & 0 & 0 & 0 & 0 & 0 & 0 & 0 & 0 & 0 & 0 & 0 & 0 & 0 & 0 & 0 & 0 & 0 & 0 & 0 & 0 & 0 & 1 & 1 & 1 & 1 & 0 & 0 & 1 & 1 & 1 & 0\end{array}$

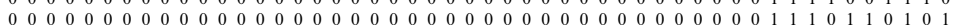
$\begin{array}{lllllllllllllllllllllllllllllllllllllllllllllllllllll}0 & 0 & 0 & 0 & 0 & 0 & 0 & 0 & 0 & 0 & 0 & 0 & 0 & 0 & 0 & 0 & 0 & 0 & 0 & 0 & 0 & 0 & 0 & 0 & 0 & 0 & 0 & 0 & 0 & 0 & 0 & 0 & 0 & 0 & 0 & 0 & 0 & 0 & 0 & 0 & 1 & 0 & 0 & 1 & 1 & 1 & 1 & 0 & 1 & 1 & 1\end{array}$ $\begin{array}{lllllllllllllllllllllllllllllllllllllllllllllllll}0 & 0 & 0 & 0 & 0 & 0 & 0 & 0 & 0 & 0 & 0 & 0 & 0 & 0 & 0 & 0 & 0 & 0 & 0 & 0 & 0 & 0 & 0 & 0 & 0 & 0 & 0 & 0 & 0 & 0 & 0 & 0 & 0 & 0 & 0 & 0 & 0 & 0 & 0 & 0 & 1 & 1 & 1 & 1 & 0 & 1 & 0 & 1 & 0\end{array}$ $\left(\begin{array}{llllllllllllllllllllllllllllllllllllllllllllllllll}0 & 0 & 0 & 0 & 0 & 0 & 0 & 0 & 0 & 0 & 0 & 0 & 0 & 0 & 0 & 0 & 0 & 0 & 0 & 0 & 0 & 0 & 0 & 0 & 0 & 0 & 0 & 0 & 0 & 0 & 0 & 0 & 0 & 0 & 0 & 0 & 0 & 0 & 0 & 0 & 0 & 1 & 1 & 1 & 1 & 1 & 0 & 1 & 1 & 1\end{array}\right]$ 
$\left(\begin{array}{llllllllllllllllllllllllllllllllllllllllllllllllll}0 & 0 & 1 & 1 & 1 & 1 & 1 & 1 & 0 & 0 & 0 & 0 & 0 & 0 & 0 & 0 & 0 & 0 & 0 & 0 & 0 & 0 & 0 & 0 & 0 & 0 & 0 & 0 & 0 & 0 & 0 & 0 & 0 & 0 & 0 & 0 & 0 & 0 & 0 & 0 & 0 & 0 & 0 & 0 & 0 & 0 & 0 & 0 & 1 & 0 \\ 0 & 0 & 1 & 1 & 1\end{array}\right.$ $\left(\begin{array}{lllllllllllllllllllllllllllllllllllllllllllllllllll}0 & 0 & 1 & 1 & 1 & 1 & 1 & 1 & 0 & 0 & 0 & 0 & 0 & 0 & 0 & 0 & 0 & 0 & 0 & 0 & 0 & 0 & 0 & 0 & 0 & 0 & 0 & 0 & 0 & 0 & 0 & 0 & 0 & 0 & 0 & 0 & 0 & 0 & 0 & 0 & 0 & 0 & 0 & 0 & 0 & 0 & 0 & 0 & 0 & 1\end{array}\right.$ $\begin{array}{llllllllllllllllllllllllllllllllllllllllllllllllll}1 & 1 & 0 & 1 & 1 & 1 & 1 & 1 & 0 & 0 & 0 & 0 & 0 & 0 & 0 & 0 & 0 & 0 & 0 & 0 & 0 & 0 & 0 & 0 & 0 & 0 & 0 & 0 & 0 & 0 & 0 & 0 & 0 & 0 & 0 & 0 & 0 & 0 & 0 & 0 & 0 & 0 & 0 & 0 & 0 & 0 & 0 & 0 & 0 & 0\end{array}$ $\begin{array}{llllllllllllllllllllllllllllllllllllllllllllllllll}1 & 1 & 1 & 0 & 1 & 1 & 1 & 1 & 0 & 0 & 0 & 0 & 0 & 0 & 0 & 0 & 0 & 0 & 0 & 0 & 0 & 0 & 0 & 0 & 0 & 0 & 0 & 0 & 0 & 0 & 0 & 0 & 0 & 0 & 0 & 0 & 0 & 0 & 0 & 0 & 0 & 0 & 0 & 0 & 0 & 0 & 0 & 0 & 0 & 0\end{array}$ $\begin{array}{lllllllllllllllllllllllllllllllllllllllllllllllllll}1 & 1 & 1 & 1 & 0 & 1 & 1 & 1 & 0 & 0 & 0 & 0 & 0 & 0 & 0 & 0 & 0 & 0 & 0 & 0 & 0 & 0 & 0 & 0 & 0 & 0 & 0 & 0 & 0 & 0 & 0 & 0 & 0 & 0 & 0 & 0 & 0 & 0 & 0 & 0 & 0 & 0 & 0 & 0 & 0 & 0 & 0 & 0 & 0 & 0\end{array}$

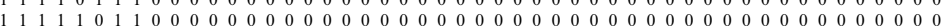
$\begin{array}{lllllllllllllllllllllllllllllllllllllllllllllllllllll}1 & 1 & 1 & 1 & 1 & 1 & 0 & 1 & 0 & 0 & 0 & 0 & 0 & 0 & 0 & 0 & 0 & 0 & 0 & 0 & 0 & 0 & 0 & 0 & 0 & 0 & 0 & 0 & 0 & 0 & 0 & 0 & 0 & 0 & 0 & 0 & 0 & 0 & 0 & 0 & 0 & 0 & 0 & 0 & 0 & 0 & 0 & 0 & 0 & 0\end{array}$

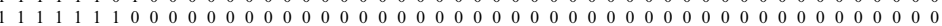
$\begin{array}{lllllllllllllllllllllllllllllllllllllllllllllllllll}0 & 0 & 0 & 0 & 0 & 0 & 0 & 0 & 0 & 0 & 1 & 1 & 1 & 1 & 1 & 1 & 0 & 0 & 0 & 0 & 0 & 0 & 0 & 0 & 0 & 0 & 0 & 0 & 0 & 0 & 0 & 0 & 0 & 0 & 0 & 0 & 0 & 0 & 0 & 0 & 0 & 0 & 0 & 0 & 0 & 0 & 0 & 0 & 1 & 0\end{array}$ $\begin{array}{llllllllllllllllllllllllllllllllllllllllllllllllll}0 & 0 & 0 & 0 & 0 & 0 & 0 & 0 & 0 & 0 & 1 & 1 & 1 & 1 & 1 & 1 & 0 & 0 & 0 & 0 & 0 & 0 & 0 & 0 & 0 & 0 & 0 & 0 & 0 & 0 & 0 & 0 & 0 & 0 & 0 & 0 & 0 & 0 & 0 & 0 & 0 & 0 & 0 & 0 & 0 & 0 & 0 & 0 & 0 & 1\end{array}$

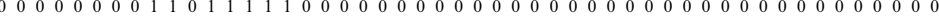

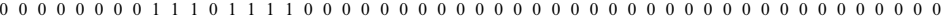
$\begin{array}{llllllllllllllllllllllllllllllllllllllllllllllllllllll}0 & 0 & 0 & 0 & 0 & 0 & 0 & 0 & 1 & 1 & 1 & 1 & 0 & 1 & 1 & 1 & 0 & 0 & 0 & 0 & 0 & 0 & 0 & 0 & 0 & 0 & 0 & 0 & 0 & 0 & 0 & 0 & 0 & 0 & 0 & 0 & 0 & 0 & 0 & 0 & 0 & 0 & 0 & 0 & 0 & 0 & 0 & 0 & 0 & 0\end{array}$

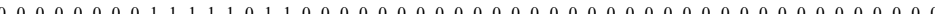
$\begin{array}{lllllllllllll}0 & 0 & 0 & 0 & 0 & 0 & 0 & 0 & 1 & 1 & 1 & 1 & 1\end{array}$ 0 $\begin{array}{llllllllllllllllllllllllllllllllllllllllllllllllll}0 & 0 & 0 & 0 & 0 & 0 & 0 & 0 & 1 & 1 & 1 & 1 & 1 & 1 & 1 & 0 & 0 & 0 & 0 & 0 & 0 & 0 & 0 & 0 & 0 & 0 & 0 & 0 & 0 & 0 & 0 & 0 & 0 & 0 & 0 & 0 & 0 & 0 & 0 & 0 & 0 & 0 & 0 & 0 & 0 & 0 & 0 & 0 & 0 & 0\end{array}$ $\begin{array}{llllllllllllllllllllllllllllllllllllllllllllllllll}0 & 0 & 0 & 0 & 0 & 0 & 0 & 0 & 0 & 0 & 0 & 0 & 0 & 0 & 0 & 0 & 0 & 0 & 1 & 1 & 1 & 1 & 1 & 1 & 0 & 0 & 0 & 0 & 0 & 0 & 0 & 0 & 0 & 0 & 0 & 0 & 0 & 0 & 0 & 0 & 0 & 0 & 0 & 0 & 0 & 0 & 0 & 0 & 1 & 0\end{array}$ $\begin{array}{lllllllllllllllllllllllllllllllllllllllllllllllllll}0 & 0 & 0 & 0 & 0 & 0 & 0 & 0 & 0 & 0 & 0 & 0 & 0 & 0 & 0 & 0 & 0 & 0 & 1 & 1 & 1 & 1 & 1 & 1 & 0 & 0 & 0 & 0 & 0 & 0 & 0 & 0 & 0 & 0 & 0 & 0 & 0 & 0 & 0 & 0 & 0 & 0 & 0 & 0 & 0 & 0 & 0 & 0 & 0 & 1\end{array}$

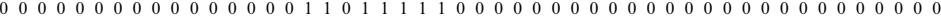
$\begin{array}{llllllllllllllllllllllllllllllllllllllllllllllllll}0 & 0 & 0 & 0 & 0 & 0 & 0 & 0 & 0 & 0 & 0 & 0 & 0 & 0 & 0 & 0 & 1 & 1 & 1 & 0 & 1 & 1 & 1 & 1 & 0 & 0 & 0 & 0 & 0 & 0 & 0 & 0 & 0 & 0 & 0 & 0 & 0 & 0 & 0 & 0 & 0 & 0 & 0 & 0 & 0 & 0 & 0 & 0 & 0 & 0\end{array}$

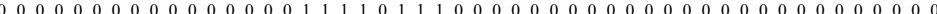

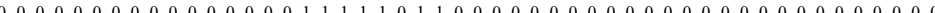

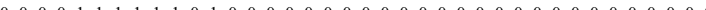
0

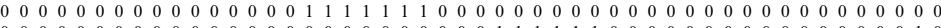
$\begin{array}{lllllllllllllllllllllllllllllllllllllllllllllllllll}0 & 0 & 0 & 0 & 0 & 0 & 0 & 0 & 0 & 0 & 0 & 0 & 0 & 0 & 0 & 0 & 0 & 0 & 0 & 0 & 0 & 0 & 0 & 0 & 0 & 0 & 1 & 1 & 1 & 1 & 1 & 1 & 0 & 0 & 0 & 0 & 0 & 0 & 0 & 0 & 0 & 0 & 0 & 0 & 0 & 0 & 0 & 0 & 1 & 0\end{array}$ $\begin{array}{llllllllllllllllllllllllllllllllllllllllllllllllll}0 & 0 & 0 & 0 & 0 & 0 & 0 & 0 & 0 & 0 & 0 & 0 & 0 & 0 & 0 & 0 & 0 & 0 & 0 & 0 & 0 & 0 & 0 & 0 & 0 & 0 & 1 & 1 & 1 & 1 & 1 & 1 & 0 & 0 & 0 & 0 & 0 & 0 & 0 & 0 & 0 & 0 & 0 & 0 & 0 & 0 & 0 & 0 & 0 & 1\end{array}$

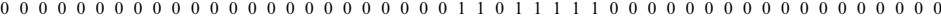
$\begin{array}{lllllllllllllllllllllllllllllllllllllllllllllllllll}0 & 0 & 0 & 0 & 0 & 0 & 0 & 0 & 0 & 0 & 0 & 0 & 0 & 0 & 0 & 0 & 0 & 0 & 0 & 0 & 0 & 0 & 0 & 0 & 1 & 1 & 1 & 0 & 1 & 1 & 1 & 1 & 0 & 0 & 0 & 0 & 0 & 0 & 0 & 0 & 0 & 0 & 0 & 0 & 0 & 0 & 0 & 0 & 0 & 0\end{array}$ $\begin{array}{llllllllllllllllllllllllllllllllllllllllllllllllllll}0 & 0 & 0 & 0 & 0 & 0 & 0 & 0 & 0 & 0 & 0 & 0 & 0 & 0 & 0 & 0 & 0 & 0 & 0 & 0 & 0 & 0 & 0 & 0 & 1 & 1 & 1 & 1 & 0 & 1 & 1 & 1 & 0 & 0 & 0 & 0 & 0 & 0 & 0 & 0 & 0 & 0 & 0 & 0 & 0 & 0 & 0 & 0 & 0 & 0\end{array}$

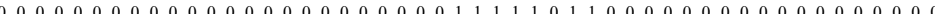
$\begin{array}{llllllllllllllllllllllllllllllllllllllllllllllllll}0 & 0 & 0 & 0 & 0 & 0 & 0 & 0 & 0 & 0 & 0 & 0 & 0 & 0 & 0 & 0 & 0 & 0 & 0 & 0 & 0 & 0 & 0 & 0 & 1 & 1 & 1 & 1 & 1 & 1 & 1 & 0 & 0 & 0 & 0 & 0 & 0 & 0 & 0 & 0 & 0 & 0 & 0 & 0 & 0 & 0 & 0 & 0 & 0 & 0 \\ 0 & 0 & 0 & 0\end{array}$ $\begin{array}{llllllllllllllllllllllllllllllllllllllllllllllllll}0 & 0 & 0 & 0 & 0 & 0 & 0 & 0 & 0 & 0 & 0 & 0 & 0 & 0 & 0 & 0 & 0 & 0 & 0 & 0 & 0 & 0 & 0 & 0 & 0 & 0 & 0 & 0 & 0 & 0 & 0 & 0 & 0 & 0 & 1 & 1 & 1 & 1 & 1 & 1 & 0 & 0 & 0 & 0 & 0 & 0 & 0 & 0 & 1 & 0\end{array}$ $\begin{array}{llllllllllllllllllllllllllllllllllllllllllllllllll}0 & 0 & 0 & 0 & 0 & 0 & 0 & 0 & 0 & 0 & 0 & 0 & 0 & 0 & 0 & 0 & 0 & 0 & 0 & 0 & 0 & 0 & 0 & 0 & 0 & 0 & 0 & 0 & 0 & 0 & 0 & 0 & 0 & 0 & 1 & 1 & 1 & 1 & 1 & 1 & 0 & 0 & 0 & 0 & 0 & 0 & 0 & 0 & 0 & 1\end{array}$ $\begin{array}{llllllllllllllllllllllllllllllllllllllllllllllllll}0 & 0 & 0 & 0 & 0 & 0 & 0 & 0 & 0 & 0 & 0 & 0 & 0 & 0 & 0 & 0 & 0 & 0 & 0 & 0 & 0 & 0 & 0 & 0 & 0 & 0 & 0 & 0 & 0 & 0 & 0 & 0 & 1 & 1 & 0 & 1 & 1 & 1 & 1 & 1 & 0 & 0 & 0 & 0 & 0 & 0 & 0 & 0 & 0 & 0\end{array}$ $\begin{array}{lllllllllllllllllllllllllllllllllllllllllllllllllll}0 & 0 & 0 & 0 & 0 & 0 & 0 & 0 & 0 & 0 & 0 & 0 & 0 & 0 & 0 & 0 & 0 & 0 & 0 & 0 & 0 & 0 & 0 & 0 & 0 & 0 & 0 & 0 & 0 & 0 & 0 & 0 & 1 & 1 & 1 & 0 & 1 & 1 & 1 & 1 & 0 & 0 & 0 & 0 & 0 & 0 & 0 & 0 & 0 & 0\end{array}$ $\begin{array}{llllllllllllllllllllllllllllllllllllllllllllllllll}0 & 0 & 0 & 0 & 0 & 0 & 0 & 0 & 0 & 0 & 0 & 0 & 0 & 0 & 0 & 0 & 0 & 0 & 0 & 0 & 0 & 0 & 0 & 0 & 0 & 0 & 0 & 0 & 0 & 0 & 0 & 0 & 1 & 1 & 1 & 1 & 0 & 1 & 1 & 1 & 0 & 0 & 0 & 0 & 0 & 0 & 0 & 0 & 0 & 0\end{array}$ $\begin{array}{llllllllllllllllllllllllllllllllllllllllllllllllllll}0 & 0 & 0 & 0 & 0 & 0 & 0 & 0 & 0 & 0 & 0 & 0 & 0 & 0 & 0 & 0 & 0 & 0 & 0 & 0 & 0 & 0 & 0 & 0 & 0 & 0 & 0 & 0 & 0 & 0 & 0 & 0 & 1 & 1 & 1 & 1 & 1 & 0 & 1 & 1 & 0 & 0 & 0 & 0 & 0 & 0 & 0 & 0 & 0 & 0\end{array}$

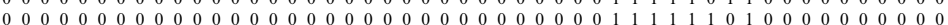

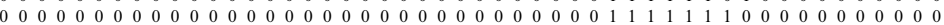
$\begin{array}{llllllllllllllllllllllllllllllllllllllllllllllllll}0 & 0 & 0 & 0 & 0 & 0 & 0 & 0 & 0 & 0 & 0 & 0 & 0 & 0 & 0 & 0 & 0 & 0 & 0 & 0 & 0 & 0 & 0 & 0 & 0 & 0 & 0 & 0 & 0 & 0 & 0 & 0 & 0 & 0 & 0 & 0 & 0 & 0 & 0 & 0 & 0 & 0 & 1 & 1 & 1 & 1 & 1 & 1 & 1 & 0\end{array}$ $\begin{array}{llllllllllllllllllllllllllllllllllllllllllllllllll}0 & 0 & 0 & 0 & 0 & 0 & 0 & 0 & 0 & 0 & 0 & 0 & 0 & 0 & 0 & 0 & 0 & 0 & 0 & 0 & 0 & 0 & 0 & 0 & 0 & 0 & 0 & 0 & 0 & 0 & 0 & 0 & 0 & 0 & 0 & 0 & 0 & 0 & 0 & 0 & 0 & 0 & 1 & 1 & 1 & 1 & 1 & 1 & 0 & 1\end{array}$ $\begin{array}{llllllllllllllllllllllllllllllllllllllllllllllllll}0 & 0 & 0 & 0 & 0 & 0 & 0 & 0 & 0 & 0 & 0 & 0 & 0 & 0 & 0 & 0 & 0 & 0 & 0 & 0 & 0 & 0 & 0 & 0 & 0 & 0 & 0 & 0 & 0 & 0 & 0 & 0 & 0 & 0 & 0 & 0 & 0 & 0 & 0 & 0 & 1 & 1 & 0 & 1 & 1 & 1 & 1 & 1 & 0 & 0\end{array}$ $\begin{array}{llllllllllllllllllllllllllllllllllllllllllllllllll}0 & 0 & 0 & 0 & 0 & 0 & 0 & 0 & 0 & 0 & 0 & 0 & 0 & 0 & 0 & 0 & 0 & 0 & 0 & 0 & 0 & 0 & 0 & 0 & 0 & 0 & 0 & 0 & 0 & 0 & 0 & 0 & 0 & 0 & 0 & 0 & 0 & 0 & 0 & 0 & 1 & 1 & 1 & 0 & 1 & 1 & 1 & 1 & 0 & 0\end{array}$

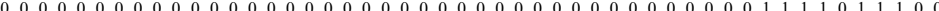

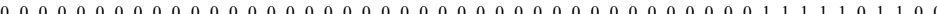

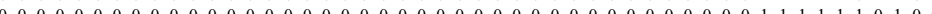
$\begin{array}{llllllllllllllllllllllllllllllllllllllllllllllllllll}0 & 0 & 0 & 0 & 0 & 0 & 0 & 0 & 0 & 0 & 0 & 0 & 0 & 0 & 0 & 0 & 0 & 0 & 0 & 0 & 0 & 0 & 0 & 0 & 0 & 0 & 0 & 0 & 0 & 0 & 0 & 0 & 0 & 0 & 0 & 0 & 0 & 0 & 0 & 0 & 1 & 1 & 1 & 1 & 1 & 1 & 1 & 0 & 0 & 0\end{array}$ $\begin{array}{llllllllllllllllllllllllllllllllllllllllllllllllllll}1 & 0 & 0 & 0 & 0 & 0 & 0 & 0 & 1 & 0 & 0 & 0 & 0 & 0 & 0 & 0 & 1 & 0 & 0 & 0 & 0 & 0 & 0 & 0 & 1 & 0 & 0 & 0 & 0 & 0 & 0 & 0 & 1 & 0 & 0 & 0 & 0 & 0 & 0 & 0 & 1 & 0 & 0 & 0 & 0 & 0 & 0 & 0 & 0 & 1\end{array}$

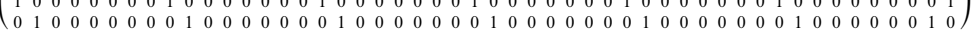

